\title{
The HC-Pro and P3 Cistrons of an Avirulent Soybean mosaic virus Are Recognized by Different Resistance Genes at the Complex Rsv1 Locus
}

\author{
R.-H. Wen, ${ }^{1}$ B. Khatabi, ${ }^{1}$ T. Ashfield, ${ }^{2}$ M. A. Saghai Maroof, ${ }^{3}$ and M. R. Hajimorad ${ }^{1}$ \\ ${ }^{1}$ Department of Entomology and Plant Pathology, The University of Tennessee, Knoxville 37996, U.S.A.; ${ }^{2}$ Department of \\ Biology, Indiana University, Bloomington 47405, U.S.A.; ${ }^{3}$ Department of Crop and Soil Environmental Sciences, Virginia \\ Tech, Blacksburg 24061, U.S.A.
}

Submitted 18 June 2012. Accepted 20 September 2012.

\begin{abstract}
The complex Rsv1 locus in soybean plant introduction (PI) 'PI96983' confers extreme resistance (ER) against Soybean mosaic virus (SMV) strain $\mathrm{N}$ but not SMV-G7 and SMVG7d. Both the SMV helper-component proteinase (HC-Pro) and $P 3$ cistrons can serve as avirulence factors recognized by Rsv1. To understand the genetics underlying recognition of the two cistrons, we have utilized two soybean lines (L800 and L943) derived from crosses between PI96983 (Rsv1) and Lee68 (rsv1) with distinct recombination events within the Rsv1 locus. L800 contains a single PI96983derived member $(3 g G 2)$ of an Rsv1-associated subfamily of nucleotide-binding leucine-rich repeat (NB-LRR) genes. In contrast, although L943 lacks $3 g G 2$, it contains a suite of five other NB-LRR genes belonging to the same family. L800 confers ER against SMV-N whereas L943 allows limited replication at the inoculation site. SMV-N-derived chimeras containing HC-Pro from SMV-G7 or SMV-G7d gained virulence on L943 but not on L800 whereas those with P3 replacement gained virulence on $\mathrm{L800}$ but not on L943. In reciprocal experiments, SMV-G7- and SMV-G7dderived chimeras with HC-Pro replacement from SMV-N lost virulence on $\mathrm{L943}$ but retained virulence on $\mathrm{L800}$ whereas those with P3 replacement lost virulence on L800 while remaining virulent on $\mathrm{L943}$. These data demonstrate that distinct resistance genes at the Rsv1 locus, likely belonging to the NB-LRR class, mediate recognition of $\mathrm{HC}$ Pro and P3.
\end{abstract}

In soybean, the Rsvl locus in plant introduction (PI) 'PI96983' confers extreme resistance (ER) against the majority of Soybean mosaic virus (SMV) strains, including SMV-N (Cho and Goodman 1979; Hajimorad and Hill 2001). However, naturally occurring SMV-G7 and its experimentally derived variant, SMV-G7d, overcome $R s v 1$-mediated ER

\section{R.-H. Wen and B. Khatabi contributed equally to this study.}

Current address of R.-H. Wen: Guangxi Key Laboratory of Subtropical Bioresource Conservation and Utilization, College of Life Sciences and Technology, Guangxi University, Nanning, Guangxi, 530004, China.

Corresponding author: M. R. Hajimorad; Fax: +1.865.974.4744; E-mail: mrh@utk.edu

* The $e$-Xtra logo stands for "electronic extra" and indicates two supplementary tables are published online.

C 2013 The American Phytopathological Society
(Hajimorad et al. 2003). Thus, in interactions with the Rsv1genotype soybean, SMV-N is avirulent (avr) while both SMVG7 and SMV-G7d are virulent (vir). The phenotype of the interaction of avrSMV-N with the Rsvl-genotype soybean is symptomless and the virus cannot be recovered from the inoculated plants (Hajimorad and Hill 2001). Furthermore, in recently reported microarray expression profiling, it was not possible to correlate $R s v 1$-mediated ER with any significant changes in gene expression (Zhang et al. 2012). However, when introduced from infected $r s v l$ stocks or scions, avrSMV-N induces limited restricted necrosis, bearing all the hallmarks characteristic of the salicylic acid-mediated active resistance pathway in PI96983 (Rsv1) (Hajimorad and Hill 2001). On the other hand, interactions of virSMV-G7 and virSMV-G7d with $R s v 1$-genotype soybean resulted in expression of lethal systemic hypersensitive response (HR) and systemic mosaic, respectively (Hajimorad et al. 2003, 2005). Induction of Rsv1mediated systemic HR by virSMV-G7 likely represents a weak recognition of the virus. Currently, the underlying molecular and biochemical mechanisms leading to induction of systemic $\mathrm{HR}$, instead of restricted localized HR, by viruses are not understood. It has been suggested that systemic HR is an indicative of "weak elicitor" function of an avirulence gene (Culver et al. 1991) or is a consequence of delayed induction of the biochemical and physiological events that are associated with localized HR (Dinesh-Kumar et al. 2000).

SMV, a single-stranded positive-sense RNA virus, is a species within the genus Potyvirus belonging to the family of Potyviridae. Its genome, approximately $9.6 \mathrm{~kb}$, contains a long open reading frame (ORF) and a small overlapping ORF known as pipo (Adams et al. 2005; Chung et al. 2008; Wen and Hajimorad 2010). Upon expression, the resultant single large polypeptide is processed posttranslationally by three viral-encoded proteinases to yield a number of multifunctional proteins, including helper-component proteinase (HC-Pro), P3, and cytoplasmic inclusion $(\mathrm{CI})$ proteins (Urcuqui-Inchima et al. 2001). The small ORF embedded in the P3 cistron (i.e., pipo) has the potential to encode a protein in the +2 frame in relation to the polyprotein ORF, and plays a role in virus movement (Chung et al. 2008; Vijayapalani et al. 2012; Wen and Hajimorad 2010).

To identify the SMV-derived elicitors of Rsv1, three different genetic approaches were previously utilized: i) comparative genomic analysis of avrSMV-N and virSMV-G7 (Eggenberger et al. 2008), ii) generation and experimental adaptation of avrSMV-N-derived chimeras containing P3 sequences from virSMV-G7 or virSMV-G7d (Hajimorad et al. 2006, 2008), 
and iii) direct adaptation of avrSMV-N to virulence on Rsv1genotype soybean via an experimental evolutionary approach (Hajimorad et al. 2011). These experiments revealed that HC-Pro and P3 serve as the SMV elicitors of Rsv1-mediated resistance. Interestingly, SMV strain-specific P3 has also been identified as the elicitor of $R s v 1$-mediated lethal systemic HR (Hajimorad et al. 2005). The P3 cistron of SMV, similar to those of other potyviruses, contains pipo; however, the involvement of PIPO protein in triggering resistance in $R s v 1$-genotype soybean has recently been ruled out (Wen et al. 2011).

The involvement of both HC-Pro and P3 in the recognition of SMV on $R s v 1$-genotype soybean remains a puzzle, and a number of hypotheses have been put forward. One hypothesis suggested that the entire polyprotein precursor or a polypeptide containing both HC-Pro and P3 may act as the SMV elicitor of Rsvl (Hajimorad et al. 2008), assuming that Rsvl-mediated recognition involves direct interaction with the SMV elicitor. However, Eggenberger and associates (2008) suggested that HC-Pro and P3 may interact with a common soybean factor, which is "guarded" (Dangl and Jones 2001) by Rsv1, analogous to the recognition of $A v r B$ and AvrRpml by the Arabidopsis RPM1 gene (Mackey et al. 2002); or, alternatively, that more than one resistance gene against SMV exists within the complex Rsvl locus region, where one gene recognizes HCPro while another detects P3. In fact, Hayes and associates (2004) have shown that the resistance provided by the Rsvl locus is multigenic. They screened a large segregating population of 1,056 individuals from a cross between PI96983 (Rsvl) and SMV-susceptible 'Lee68' ( $r s v 1)$ and subsequently identified unique soybean recombinant inbred lines (RIL) with specific recombination events within the complex $R s v 1$ locus.
One such RIL (L800) contains only a single PI96983-derived member, " $3 g G 2$ ", of a subfamily (the class G family) of nucleotide-binding leucine-rich repeat (NB-LRR) genes shown to be closely associated with the Rsvl locus on molecular linkage group MLG-F (chromosome 13) (Hayes et al. 2004). L800 (3gG2), similar to PI96983 (Rsv1), confers ER against avrSMV-N but not against virSMV-G7 and vir SMV-G7d (Hajimorad et al. 2011; Wen et al. 2011). The $3 g G 2$ ORF
$\mathrm{N}$

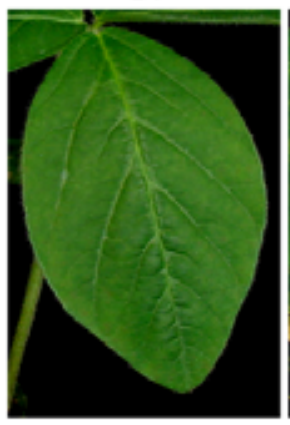

G7

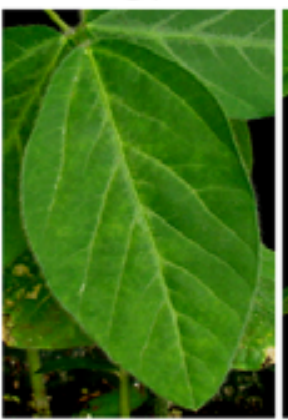

G7d

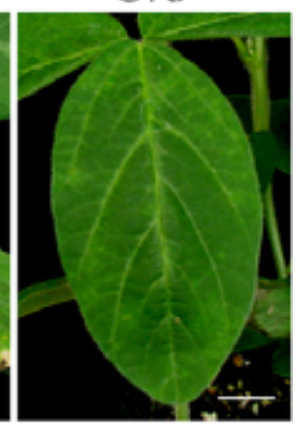

Fig. 2. Phenotypic responses of recombinant inbred soybean line L943 $(-3 g G 2)$ to mechanical inoculation with Soybean mosaic virus (SMV) strains avirulent (avr)SMV-N (N), virulent (vir)SMV-G7 (G7), and virSMV-G7d (G7d). Sap containing progeny viruses derived from 'Williams82' $(r s v 1)$ biolistically inoculated with infectious cDNA clones served as inoculum. Inoculated plants were maintained at $22^{\circ} \mathrm{C}$ until leaflets from trifoliate leaves were photographed at 21 days postinoculation. Note that plants inoculated with avrSMV-N, in contrast to those inoculated with virSMV-G7 and virSMV-G7d that developed mild systemic mosaic, remained asymptomatic and the virus could not be detected from the noninoculated leaves by enzyme-linked immunosorbent assay. Scale bar $=1 \mathrm{~cm}$.

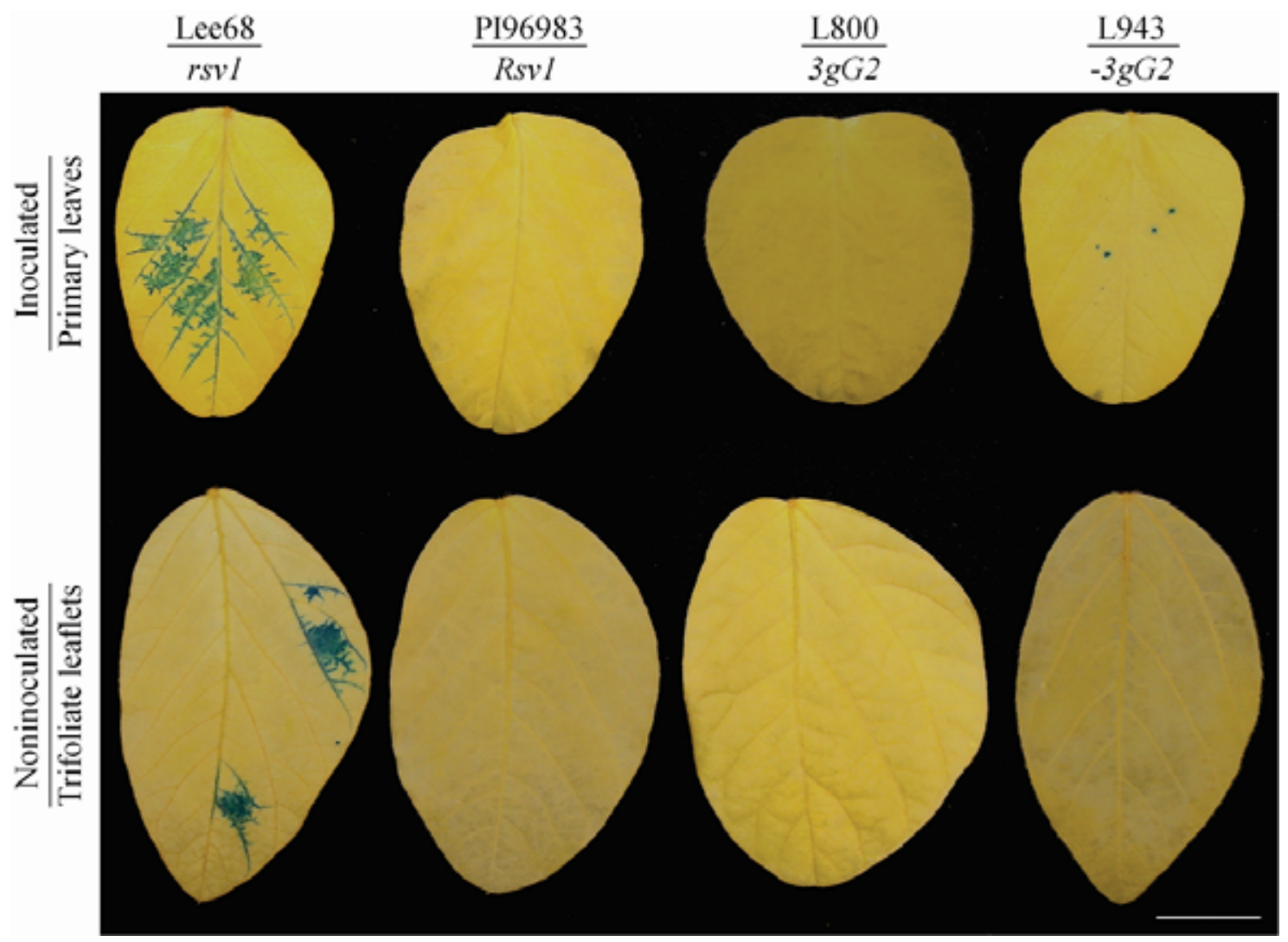

Fig. 1. $\beta$-Glucuronidase (GUS)-histochemical analysis of Soybean mosaic virus (SMV) infection in inoculated and noninoculated soybean leaves. For inoculation, a plasmid containing a full-length infectious cDNA clone of avrSMV-NGUS was delivered biolistically into fully expanded attached primary leaves of 2-week-old soybean seedlings maintained at $22^{\circ} \mathrm{C}$. Histochemical analysis was done at 14 days postinoculation (dpi). Note that the patterns of GUS staining for the noninoculated trifoliate leaflets at $28 \mathrm{dpi}$ were similar to those obtained at 14 dpi. Scale bar $=2 \mathrm{~cm}$. 
(GenBank accession number AY518517) is 3,390 bp in length and encodes a deduced product similar to previously cloned coiled-coil (CC)-NB-LRR class disease resistance proteins (Hayes et al. 2004). Interestingly, nonsynonymous mutations in different locations within the P3 cistron but not the HC-Pro cistron functionally converted avrSMV-N to virSMV-N on L800 (3gG2) but not on PI96983 (Rsv1), and systemically infected L800 ( $3 g G 2)$ plants displayed systemic necrosis with various severity (Hajimorad et al. 2011; Wen et al. 2011). This is despite the fact that the phenotype of interactions of avrSMV-N, virSMV-G7, and virSMV-G7d on L800 (3gG2) (Wen et al. 2011) resembles those displayed by PI96983 (Rsv1) (Hajimorad and Hill 2001; Hajimorad et al. 2003). Hayes and associates (2004) identified an additional soybean RIL (L943) that did not carry the $3 g G 2$ gene but contained a suite of five other class G CC-NB-LRR genes (IeG30, 5gG3, IeG15, 6gG9, and $I g G 4)$ from the chromosomal region of the Rsv1 locus in PI96983 (Rsv1). Two of these genes, $5 g G 3$ and $6 g G 9$, have been isolated and sequenced (accession numbers AY518518 and AY518519, respectively). Despite the lack of the $3 g G 2$ gene, L943 displayed symptomless resistance against selected avirulent strains of SMV, including an isolate belonging to the same strain group (G2) as avrSMV-N (Hayes et al. 2004). Thus, the resistance phenotypes of these two RIL, L800 (3gG2) and L943 (-3gG2), against SMV suggest that the region containing the complex Rsvl locus (hereafter referred to as the $R s v 1$ locus) contains multiple resistance factors that recognize SMV.

Our main objective in this study was to test two hypotheses: i) HC-Pro and P3 are recognized independently and ii) the Rsv1 locus consists of different resistance genes independently recognizing HC-Pro or P3. It should be noted that, for the purpose of this article, we have adopted the definition of virulence according to Shaner and associates (1992). Virulence is defined as the ability of SMV to overcome ER, or restriction in movement, thus allowing establishment of systemic infection in either PI96983 (Rsv1), L800 (3gG2), or L943 (-3gG2), regardless of the phenotypic expression. According to this definition, in addition to strains, or variants, that cause systemic mosaic, an SMV strain such as SMV-G7 that overcomes Rsvl-mediated ER and induces systemic HR in PI96983 (Rsvl) and L800 (3gG2) is also considered virulent on these soybean genotypes. Although the expression of systemic HR is generally considered to be indicative of weak recognition, the primary role of HR in some viral pathosystems is questionable (Bendahmane et al. 1999; Hajimorad and Hill 2001). In this study, a gain in the ability of an SMV strain, or a variant, to establish systemic infection is simply used to infer a significant reduction in the efficiency of resistance $(R)$-gene-mediated recognition.

\section{RESULTS}

Phenotypic responses of L943 (-3gG2) and L800 (3gG2) to avrSMV-N, virSMV-G7, and virSMV-G7d differ.

When unifoliate leaves of 2-week-old seedlings were biolistically inoculated with avrSMV-N- $\beta$-glucuronidase (GUS), those from L943 $(-3 g G 2)$ allowed limited virus replication, as was evident by a few small clusters of cells expressing GUS (Fig. 1). However, the foci of infection remained restricted and did not extend to veins, and the virus failed to move systemically because no GUS expression was detected in the noninoculated trifoliate leaves by 28 days postinoculation (dpi) (Fig. 1). In contrast, L800 (3gG2)-inoculated leaves, like the parental Rsv1-genotype soybean PI96983, exhibited ER to avrSMV-N, as was evident by lack of any GUS expression in the inoculated primary leaves as well as noninoculated trifoliate leaflets (Fig. 1). As expected, the susceptible Lee68 ( $r s v 1$ ) supported the replication and systemic movement of avrSMV-N-GUS,

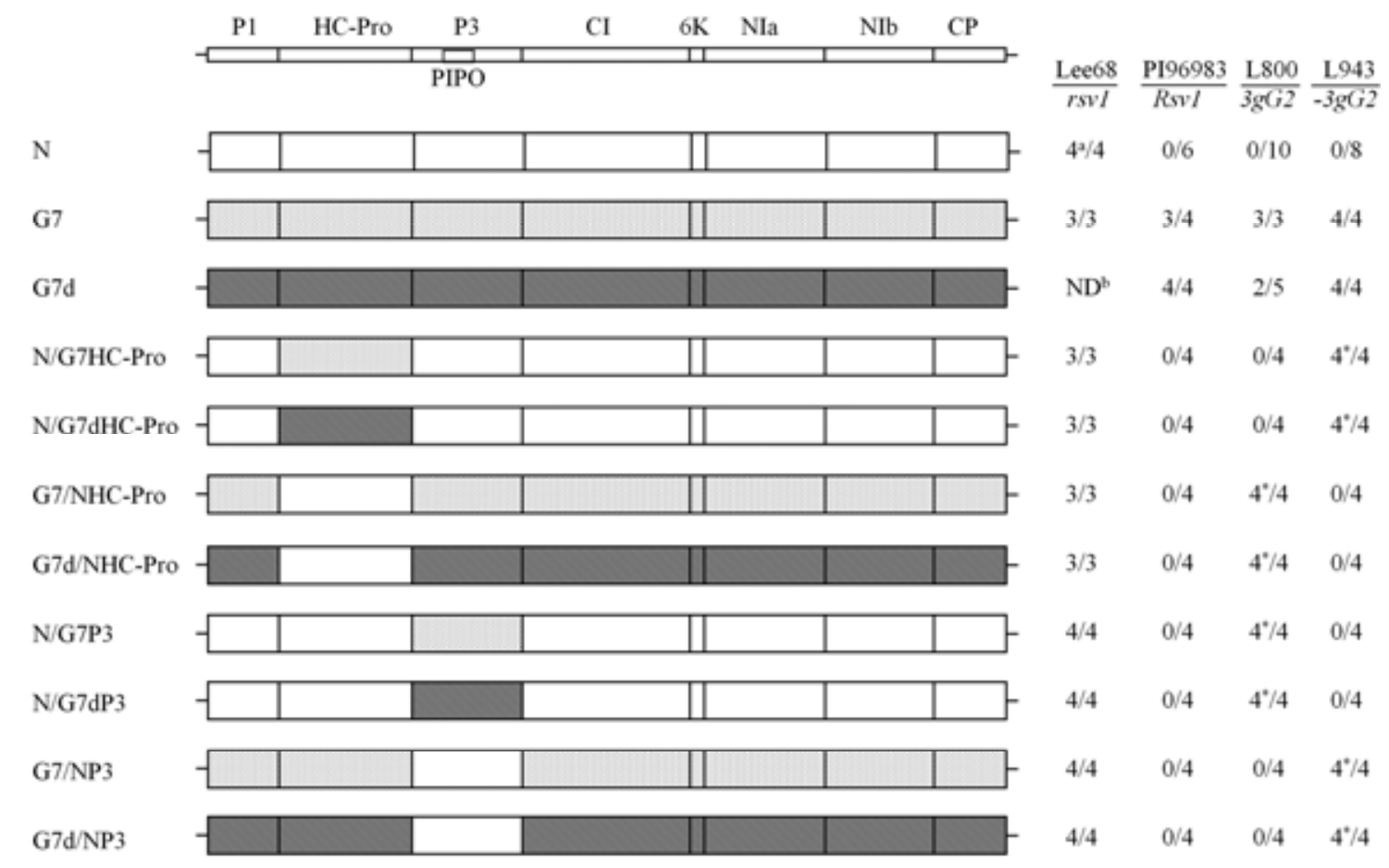

Fig. 3. Schematic representation of the genomes of Soybean mosaic virus (SMV); parental strains avirulent (avr)SMV-N (N), virulent (vir)SMV-G7 (G7), and virSMV-G7d (G7d); and their derivative chimeras with precise exchanges of helper-component proteinase (HC-Pro) or P3. The ability of the viruses to systemically infect biolistically inoculated soybean genotypes maintained at $22^{\circ} \mathrm{C}$ are indicated at the right side. The inoculated plants were evaluated for infection based on symptom expression at 28 days postinoculation. Absence of virus in asymptomatic plants was confirmed by enzyme-linked immunosorbent assay. Superscripts: $a=$ number of plants systemically infected/number of plants inoculated; $b=$ not done; $*$ indicates that the HC-Pro and P3 cistrons of viral progenies derived from one of the infected plants were reverse-transcriptase polymerase chain reaction amplified and directly sequenced. These analyses confirmed the absence of any in planta-generated mutation in either the HC-Pro or P3 cistrons. 
which was evident by efficient GUS expression in both inoculated and noninoculated leaves (Fig. 1). It should be noted that GUS expression in the inoculated primary leaves of L943 $(-3 g G 2)$ was not due to the transient expression of biolistically delivered plasmids containing the GUS gene because no GUS expression was evident in the inoculated leaves of L800 (3gG2) and PI96983 (Rsvl) under the same experimental conditions (Fig. 1).

Phenotypic responses of L800 (3gG2) plants to inoculation with virSMV-G7 and virSMV-G7d, similar to the parental $R s v 1$-genotype soybean PI96983 (Rsvl) are expressed as sys- temic HR and systemic mosaic, respectively (Hajimorad et al. 2003, 2005; Wen et al. 2011). In contrast, inoculation of L943 $(-3 g G 2)$ with virSMV-G7 or virSMV-G7d resulted in the expression of mild systemic mosaic by both viruses whereas those plants inoculated with avrSMV-N remained symptomless (Fig. 2).

\section{The HC-Pro cistron of avrSMV-N acts as the avirulence factor on L943 (-3gG2) but not on L800 (3gG2).}

It has been well established that virulence of SMV on Rsv1genotype soybean, including PI96983, is determined by strain-

Table 1. Ability of Soybean mosaic virus (SMV) strain N-derived mutants to infect soybean genotypes systemically ${ }^{\mathrm{a}}$

\begin{tabular}{|c|c|c|c|c|}
\hline Viruses $^{b}$ & Lee68 (rsv1) & PI96983 (Rsv1) & $\mathrm{L800}(3 g G 2)$ & L943 (-3gG2) \\
\hline \multicolumn{5}{|l|}{ HC-Pro Mutants } \\
\hline SMV-N $\mathrm{F}_{\mathrm{F} 16 \mathrm{~S}}$ & $7 / 7$ & $0 / 48$ & $0 / 4$ & $5 * / 9$ \\
\hline SMV-N $N_{F 317 S}$ & $13 / 13$ & $0 / 43$ & $0 / 4$ & $28 * / 28$ \\
\hline $\mathrm{SMV}-\mathrm{N}_{\mathrm{R} 318 \mathrm{P}}$ & $7 / 7$ & $0 / 4$ & $1 * / 9$ & $5 * / 5$ \\
\hline SMV-N ${ }_{G 319 D}$ & $7 / 7$ & $0 / 47$ & $0 / 4$ & $10 * / 10$ \\
\hline $\mathrm{SMV}-\mathrm{N}_{\mathrm{G} 319 \mathrm{~S}}$ & $11 / 11$ & $0 / 11$ & $0 / 4$ & $23 * / 23$ \\
\hline $\mathrm{SMV}-\mathrm{N}_{\mathrm{W} 320 \mathrm{~L}}$ & $7 / 7$ & $0 / 10$ & $0 / 11$ & $7 * / 7$ \\
\hline $\mathrm{SMV}-\mathrm{N}_{\mathrm{K} 321 \mathrm{E}}$ & $11 / 11$ & $0 / 4$ & $1 * / 12$ & $1 * / 22$ \\
\hline $\mathrm{SMV}-\mathrm{N}_{\mathrm{G} 319 \mathrm{~S}+\mathrm{K} 321 \mathrm{E}}$ & $9 / 9$ & $0 / 5$ & $0 / 14$ & $17 * / 17$ \\
\hline $\mathrm{SMV}-\mathrm{N}_{\mathrm{K} 326 \mathrm{E}}$ & $7 / 7$ & $0 / 52$ & $0 / 4$ & $3 * / 8$ \\
\hline $\mathrm{SMV}-\mathrm{N}_{\mathrm{T} 341 \mathrm{I}}$ & $11 / 11$ & $0 / 33$ & $0 / 12$ & $19 * / 19$ \\
\hline SMV-N & $2 / 3$ & $0 / 5$ & $0 / 10$ & $20 * / 20$ \\
\hline SMV-N & $6 / 6$ & $0 / 47$ & $0 / 8$ & $8 * / 8$ \\
\hline $\mathrm{SMV}-\mathrm{N}_{\mathrm{C} 367 \mathrm{Y}}$ & $7 / 7$ & $0 / 44$ & $0 / 4$ & $9 * / 9$ \\
\hline SMV-N ${ }_{R 682 K}$ & $17 / 17$ & $0 / 5$ & $0 / 20$ & $37 * / 50$ \\
\hline SMV-N $\mathrm{R}_{\mathrm{R} 82 \mathrm{G}}$ & $6 / 6$ & $0 / 14$ & $0 / 8$ & $25 * / 25$ \\
\hline SMV-N $\mathrm{N}_{\mathrm{R} 62 \mathrm{M}}$ & $11 / 11$ & $0 / 13$ & $0 / 4$ & $26 * / 27$ \\
\hline \multicolumn{5}{|l|}{ P3 Mutants } \\
\hline SMV-N ${ }_{R 787 I}$ & $5 / 5$ & $0 / 5$ & $2 / 5$ & $0 / 4$ \\
\hline $\mathrm{SMV}-\mathrm{N}_{\mathrm{V} 822 \mathrm{M}}$ & $8 / 8$ & $0 / 11$ & $8 * / 8$ & $0 / 8$ \\
\hline $\mathrm{SMV}-\mathrm{N}_{\mathrm{R} 945 \mathrm{G}}$ & $4 / 4$ & $0 / 9$ & $4 / 5$ & $1 * / 4$ \\
\hline SMV-N ${ }_{\mathrm{A} 947 \mathrm{~T}}$ & $5 / 5$ & $0 / 9$ & $4 / 4$ & $0 / 5$ \\
\hline SMV-N ${ }_{\mathrm{A} 947 \mathrm{~V}}$ & $5 / 5$ & $0 / 9$ & $3 / 4$ & $0 / 19$ \\
\hline SMV-N & $3 / 4$ & $0 / 9$ & $2 / 4$ & $0 / 4$ \\
\hline $\mathrm{SMV}-\mathrm{N}_{\mathrm{K} 952 \mathrm{E}}$ & $13 / 14$ & $0 / 20$ & $3 / 20$ & $0 / 8$ \\
\hline SMV-N $\mathrm{V}_{\mathrm{V} 1045 \mathrm{~A}}$ & $5 / 5$ & $0 / 11$ & $5 / 8$ & $0 / 5$ \\
\hline SMV-N $\mathrm{A}_{\mathrm{A} 1111 \mathrm{~V}}$ & $5 / 5$ & $0 / 6$ & $1 * / 24$ & $5 * / 17$ \\
\hline SMV-N & $5 / 5$ & $0 / 10$ & $0 / 4$ & $1 * / 11$ \\
\hline 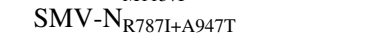 & $6 / 6$ & $1 * / 25$ & $4 / 4$ & $1 * / 4$ \\
\hline $\mathrm{SMV}-\mathrm{N}_{\mathrm{V} 822 \mathrm{M}+\mathrm{K} 952 \mathrm{E}}$ & $13 / 13$ & $0 / 19$ & $9 * / 9$ & $0 / 16$ \\
\hline $\mathrm{SMV}-\mathrm{N}_{\mathrm{V} 822 \mathrm{M}+\mathrm{K} 952 \mathrm{E}+\mathrm{A} 1111 \mathrm{~V}}$ & $5 / 5$ & $0 / 6$ & $3 * / 11$ & $0 / 7$ \\
\hline $\mathrm{SMVN}_{\mathrm{V} 822 \mathrm{M}+\mathrm{K} 952 \mathrm{E}+\mathrm{A} 1111 \mathrm{~V}+\mathrm{E} 767 \mathrm{G}}$ & $11 / 11$ & $0 / 7$ & $10 * / 10$ & $0 / 14$ \\
\hline \multicolumn{5}{|l|}{ HC-Pro + P3 Mutants } \\
\hline SMV-N ${ }_{\mathrm{K} 321 \mathrm{E}+\mathrm{A} 947 \mathrm{~V}}$ & $3 / 4$ & $0 / 9$ & $2 / 4$ & $1 * / 5$ \\
\hline $\mathrm{SMV}-\mathrm{N}_{\mathrm{K} 321 \mathrm{E}+\mathrm{R} 945 \mathrm{G}}$ & $7 / 7$ & $0 / 6$ & $5 / 5$ & $0 / 7$ \\
\hline $\mathrm{SMV}-\mathrm{N}_{\mathrm{K} 321 \mathrm{E}+\mathrm{T} 341 \mathrm{I}+\mathrm{A} 947 \mathrm{~V}}$ & $7 / 7$ & $5 / 6$ & $5 / 6$ & $13 * / 14$ \\
\hline 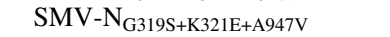 & $6 / 6$ & $5 / 6$ & $8 / 8$ & $6 * / 7$ \\
\hline $\mathrm{SMV}-\mathrm{N}_{\mathrm{R} 682 \mathrm{M}+\mathrm{R} 787 \mathrm{I}+\mathrm{A} 947 \mathrm{~T}}$ & $5 / 5$ & $6 / 6$ & $4 / 4$ & $7 * / 7$ \\
\hline
\end{tabular}

${ }^{\text {a }}$ Progeny viruses in sap extract derived from 'Williams82' ( $\left.r s v 1\right)$ biolistically inoculated with cDNA clones of the mutant viruses served as inoculum which was rub inoculated onto fully expanded primary leaves of 2-week-old soybean seedlings. Inoculated plants were maintained at $22^{\circ} \mathrm{C}$ until evaluated for infection based on symptom expression at 28 days postinoculation. Absence of virus in asymptomatic plants was confirmed by enzyme-linked immunosorbent assay. Numbers indicate number of plants systemically infected/number of plants inoculated. An asterisk (*) indicates that total RNA was extracted from one of the infected plants and subjected to reverse-transcriptase polymerase chain reaction. The stability of the mutations and absence of any newly emerged substitutions in progeny viruses was confirmed by sequencing the entire helper-component proteinase (HC-Pro) and P3 cistrons. Infection of the L800 (3gG2) plant with SMV-N $\mathrm{N}_{\mathrm{R} 318 \mathrm{P}}$ was associated with a nonsynonymous mutation resulting in A847V substitution in the P3. Infection of the L943 (-3gG2) plant with SMV-N $\mathrm{K}_{\mathrm{K} 321 \mathrm{E}}$ was associated with a nonsynonymous mutation resulting in E347V substitution in the HC-Pro. Infection of L800 (3gG2) with SMV-N $\mathrm{K}_{221 \mathrm{E}}$ was associated with a newly emerged synonymous mutation (A1271G) in HC-Pro and a sequence polymorphism in the P3 at position 2,709, such that both TCA and ACA codons (encoding serine and threonine, respectively), were present at polyprotein position 860. Infection of L943 (-3gG2) with SMV-N $\mathrm{K}_{\mathrm{K} 326 \mathrm{E}}$ was associated with a nonsynonymous mutation resulting in D339N substitution in the HC-Pro. Infection of L943 (-3gG2) with SMV-N $\mathrm{N}_{\mathrm{R} 82 \mathrm{G}}$ was associated with a synonymous mutation $(\mathrm{C} 1616 \mathrm{~T})$ in the HC-Pro. Infection of L943 (-3gG2) by SMV-N $\mathrm{R}_{\mathrm{R} 95 \mathrm{G}}$ was associated with two newly emerged nonsynonymous mutations resulting in W320L and A719V substitutions, respectively, in the HC-Pro. Infection of L943 ( $-3 g G 2)$ by SMV-N ${ }_{\text {M111v }}$ was associated with a nonsynonymous mutation which resulted in G319D substitution in the HC-Pro. Infection of L943 by SMV-N ${ }_{\text {M1157I }}$ was associated with a nonsynonymous mutation which resulted in P358S substi-

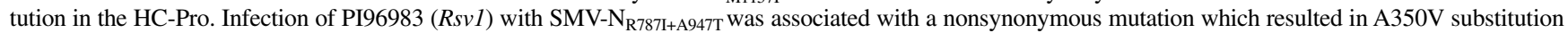

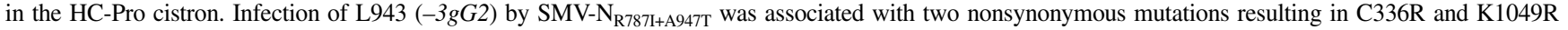
substitutions in the HC-Pro and P3, respectively. Infection of L943 (-3gG2) by SMV-N $\mathrm{K}_{\mathrm{K} 321 \mathrm{E}+\mathrm{A} 947 \mathrm{~V}}$ was associated with a nonsynonymous mutation which resulted in A350T substitution in the HC-Pro. Stability of other P3-derived mutants capable of infecting L800 (3gG2) has previously been reported (Hajimorad et al. 2011). No newly emerged mutations in the HC-Pro or P3 cistrons of the progeny viruses isolated from L800 (3gG2) or L943 ( $-3 g G 2$ ) plants infected with the other SMV-N-derived mutants were detected.

b Interactions of all the viruses with PI96983 (Rsv1) and some of them with L800 (3gG2) have previously been reported (Hajimorad et al. 2006, 2008, 2011). 
specific determinants in both the HC-Pro and P3 cistrons (Eggenberger et al. 2008; Hajimorad et al. 2008, 2011). The observation that determinants residing on $\mathrm{P} 3$ influence virulence on L800 ( $3 g G 2)$ whereas those on HC-Pro do not (Hajimorad et al. 20011; Wen et al. 2011) led us to hypothesize that strain-specific HC-Pro determines virulence on L943 $(-3 g G 2)$ but not on L800 (3gG2). To test this hypothesis, we precisely replaced the HC-Pro of avrSMV-N with those of virSMV-G7 and virSMV-G7d and vice versa (Fig. 3).

Inoculation of SMV-N/G7HC-Pro or SMV-N/G7dHC-Pro, biolistically with the infectious clones or mechanically with sap extract containing progeny viruses, resulted in gain of virulence on L943 ( $-3 g G 2)$ but not on L800 (3gG2), regardless of the method of inoculation (Fig. 3; Supplementary Table S1). Both of the chimeras induced systemic mosaic in L943 $(-3 g G 2)$ similar to virSMV-G7 and virSMV-G7d (data not shown). On the other hand, both SMV-G7/NHC-Pro and SMV-G7d/NHCPro lost virulence on L943 $(-3 g G 2)$ but remained virulent on L800 (3gG2) regardless of the methods of inoculation (Fig. 3). None of the above avrSMV-N, virSMV-G7-, or virSMV-G7dderived HC-Pro-chimeras were virulent on PI96983 (Rsvl) regardless of whether plants were inoculated biolistically with the infectious clones or mechanically using sap extract containing progeny viruses (Fig. 3). The above observations suggest that strain-specific HC-Pro of SMV determines avirulence or virulence functions on L943 $(-3 g G 2)$ but not on L800 $(3 g G 2)$. Furthermore, they confirm that both HC-Pro and P3 impact virulence on PI96983 (Rsv1) (Eggenberger et al. 2008; Hajimorad et al. 2008, 2011).

\section{Amino acid substitutions in the HC-Pro cistron of avrSMV-N alone confer virulence on L943 (-3gG2) but not on L800 (3gG2).}

Nonsynonymous mutations in the HC-Pro of avrSMV-N were previously identified that, in combination with nonsynonymous mutations in P3, were essential for gain of virulence on PI96983 (Rsvl) (Eggenberger et al. 2008; Hajimorad et al. 2008, 2011). We evaluated the impact of these HC-Pro mutations on virulence of avrSMV-N on L943 (-3gG2) and L800 (3gG2). As expected, all the HC-Pro mutants were replication competent in Lee68 (rsvl) and none were virulent on PI96983 (Rsvl) (Table 1). However, all the HC-Pro-mutants, except SMV-N $\mathrm{N}_{\mathrm{K} 321 \mathrm{E}}$, were virulent on L943 (-3gG2) (Table 1). Out of 22 L943 (-3gG2) plants inoculated with $\mathrm{SMV}-\mathrm{N}_{\mathrm{K} 321 \mathrm{E}^{-}}$derived progeny viruses, only a single plant became systemically infected; however, this was associated with a newly emerged nonsynonymous mutation in the HC-Pro resulting in E347V substitution. This mutation (K321E) in the HC-Pro cistron of avrSMV-N is interesting because, in combination with either the T341I or G319S substitutions in HC-Pro together with the A947V mutation in P3, it converted avrSMV-N to virSMV-N on PI96983 (Rsvl) or on L78-379 (Rsv1) (Table 1) (Hajimorad et al. 2011). Yet, K321E alone or in combination with A947V or R945G substitutions in the P3 does not confer virulence to avrSMV-N on L943 (-3gG2) (Table 1). Alone among the HC-Pro mutants, avr SMV-N $\mathrm{N}_{\mathrm{K} 321 \mathrm{E}}$ and avrSMV-N $\mathrm{R}_{\mathrm{R} 318 \mathrm{P}}$ were also virulent on L800 (3gG2) (Table 1). However, in both instances, the virulence of the progeny viruses was associated with newly emerged nonsynonymous mutations in the P3 (Table 1). Collectively, these observations

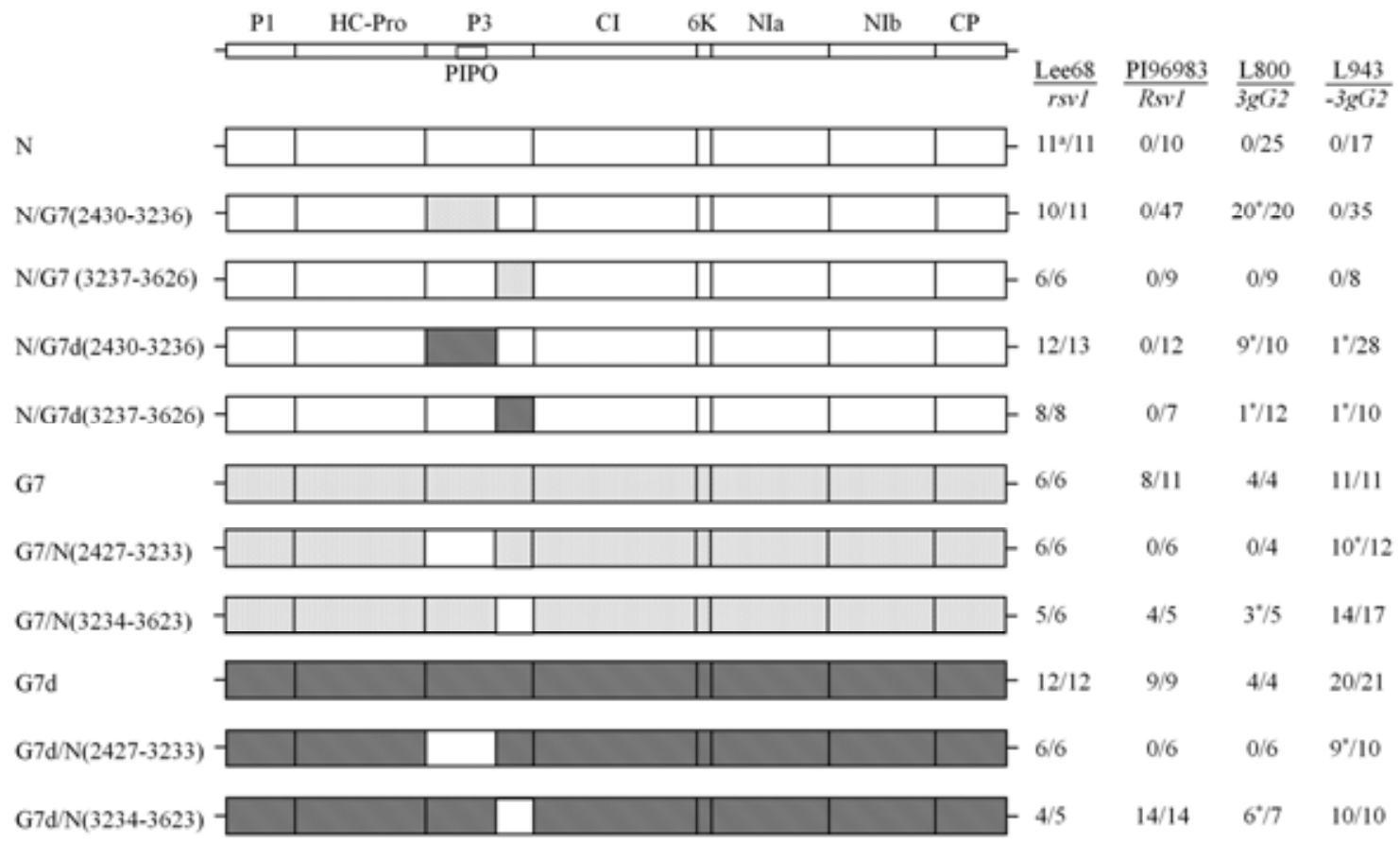

Fig. 4. Schematic representation of the genomic map of Soybean mosaic virus (SMV); parental strains avirulent (avr)SMV-N (N), virulent (vir)SMV-G7 (G7), and virSMV-G7d (G7d); and their derivative P3 chimeras. The ability of the viruses to systemically infect mechanically inoculated soybean plants kept at $22^{\circ} \mathrm{C}$ is indicated at the right side. Sap containing progeny viruses derived from 'Williams82' ( $\left.r s v 1\right)$ biolistically inoculated with infectious cDNA clones served as the inoculum. Inoculated plants were evaluated for infection based on symptom expression at 28 days postinoculation. Absence of virus in asymptomatic plants was confirmed by enzyme-linked immunosorbent assay. Superscripts: a = number of plants systemically infected/number of plants inoculated and * indicates that the helper-component proteinase (HC-Pro) and P3 cistrons of viral progenies derived from one of the infected plants were reverse-transcriptase polymerase chain reaction amplified and directly sequenced. Infection of L943 (-3gG2) with SMV-N/G7d $\mathrm{d}_{(2430-3236)}$ was associated with sequence polymorphism at nucleotide position 1,192, where glutamine and leucine were both present at amino acid position 354 within the HC-Pro cistron. Furthermore, an additional synonymous mutation, C1337T, within the HC-Pro was also associated with infection. Infection of L800 (3gG2) and L943 (-3gG2) with

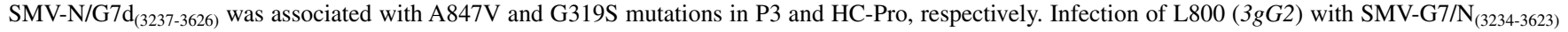
was associated with sequence polymorphism at nucleotide position 1,165, where cysteine and phenylalanine were both present at amino acid position 345 within the HC-Pro. Infection of L800 (3gG2) and L943 (-3gG2) with the progeny viruses derived from other chimeras was not associated with any mutation in either HC-Pro or P3. 
suggest that HC-Pro of avrSMV-N is recognized as the avirulence factor on L943 (-3gG2) but not on L800 (3gG2).

The $P 3$ cistron in avrSMV-N acts as an avirulence factor on L800 (3gG2) but not on L943 (-3gG2).

It has been previously shown that precise replacement of the P3 cistron in avrSMV-N with P3 from virSMV-G7 and virSMV-G7d did not result in gain of virulence by SMV-N/ G7P3 and SMV-N/G7dP3 chimeras on PI96983 (Rsv1) (Hajimorad et al. 2006). This was despite the fact that the reciprocal exchanges of the P3 cistron of virSMV-G7 and virSMV-G7d with that of avrSMV-N resulted in loss of virulence by both SMV-G7/NP3 and SMV-G7d/NP3 chimeras on PI96983 (Rsvl) (Hajimorad et al. 2006).

In contrast to the above observations on PI96983 (Rsvl), when L800 (3gG2) was inoculated with SMV-N/G7P3 or SMV-N/G7dP3, both chimeras gained virulence and caused systemic infection (Fig. 3). On the other hand, both SMV-G7/ NP3 and SMV-G7d/NP3 lost virulence on L800 (3gG2) (Fig. $3)$. This was regardless of whether plants were inoculated biolistically with the infectious cDNA clones (Fig. 3) or mechanically using sap extract containing the progeny viruses derived from the biolistically inoculated 'Williams82' ( $r s v 1)$.

Interestingly, inoculation of L943 (-3gG2) with SMVN/G7P3 or SMV-N/G7dP3, biolistically or mechanically, did not result in gain of virulence by either of the two chimeras (Fig. 3). Systemic infection of a single L943 (-3gG2) plant, out of 32 inoculated, following mechanical inoculation with progeny viruses derived from SMV-N/G7P3 was associated with two newly emerged amino acid substitutions in the HCPro. On the other hand, regardless of biolistic or mechanical inoculation, both SMV-G7/NP3 and SMV-G7d/NP3 retained virulence on L943 (-3gG2) (Fig. 3). These observations suggest that strain-specific P3 alone determines virulence or avirulence function on L800 $(3 g G 2)$ and that the Rsv1-derived genetic factors that are present in L943 $(-3 g G 2)$ target HC-Pro of avrSMV-N for recognition.

The $\mathrm{N}$-terminus of the $\mathrm{P3}$ cistron in avrSMV-N conditions avirulence on L800 (3gG2) but not on L943 (-3gG2).

The virulence determinants of SMV on PI96983 (Rsvl) residing on P3 cistron were previously mapped to the $\mathrm{N}$ terminus spanning nucleotides 2,430 to 3,236 in the genomes of virSMVG7 and virSMV-G7d (Hajimorad et al. 2006, 2008). This region of P3 corresponds to nucleotides 2,427 to 3,233 in the avrSMV-N genome, an interval that contains avirulence determinants (Hajimorad et al. 2006). The C-terminal part of P3, corresponding to nucleotides 3,237 to 3,626 in the genomes of virSMV-G7 and virSMV-G7d and nucleotides 3,234 to 3,623 in avrSMV-N, contains no primary determinants for virulence or avirulence on PI96983 (Rsv1) (Hajimorad et al. 2006).

By mechanical inoculation of L800 (3gG2) or L943 (-3gG2), we examined the virulence of progeny viruses derived from chimerical avrSMV-N, virSMV-G7, or virSMV-G7d with reciprocal exchanges of either the $\mathrm{N}$ or $\mathrm{C}$ termini of P3 (Fig. 4). Progeny viruses derived from SMV-N/G7P3 $3_{(2430-3236)}$ and SMV-N/G7dP $3_{(2430-3236)}$ were virulent on L800 (3gG2) but not on L943 $(-3 g G 2)$. On the other hand, progeny viruses derived from SMV-N/G7P3 $3_{(3237-3626)}$ and SMV-N/G7dP3 $3_{(3237-3626)}$ remained avirulent on both L800 (3gG2) and L943 (-3gG2) (Fig. 4). Progeny viruses from SMV-G7/NP3 $3_{(2427-3233)}$ and SMV-G7d/NP3 ${ }_{(2427-3233)}$ lost virulence on L800 (3gG2) but remained virulent on L943 (-3gG2) (Fig. 4). Progeny viruses derived from SMV-G7/NP3 $3_{(3237-3626)}$ or SMV-G7d/NP3 $3_{(3237-3626)}$,

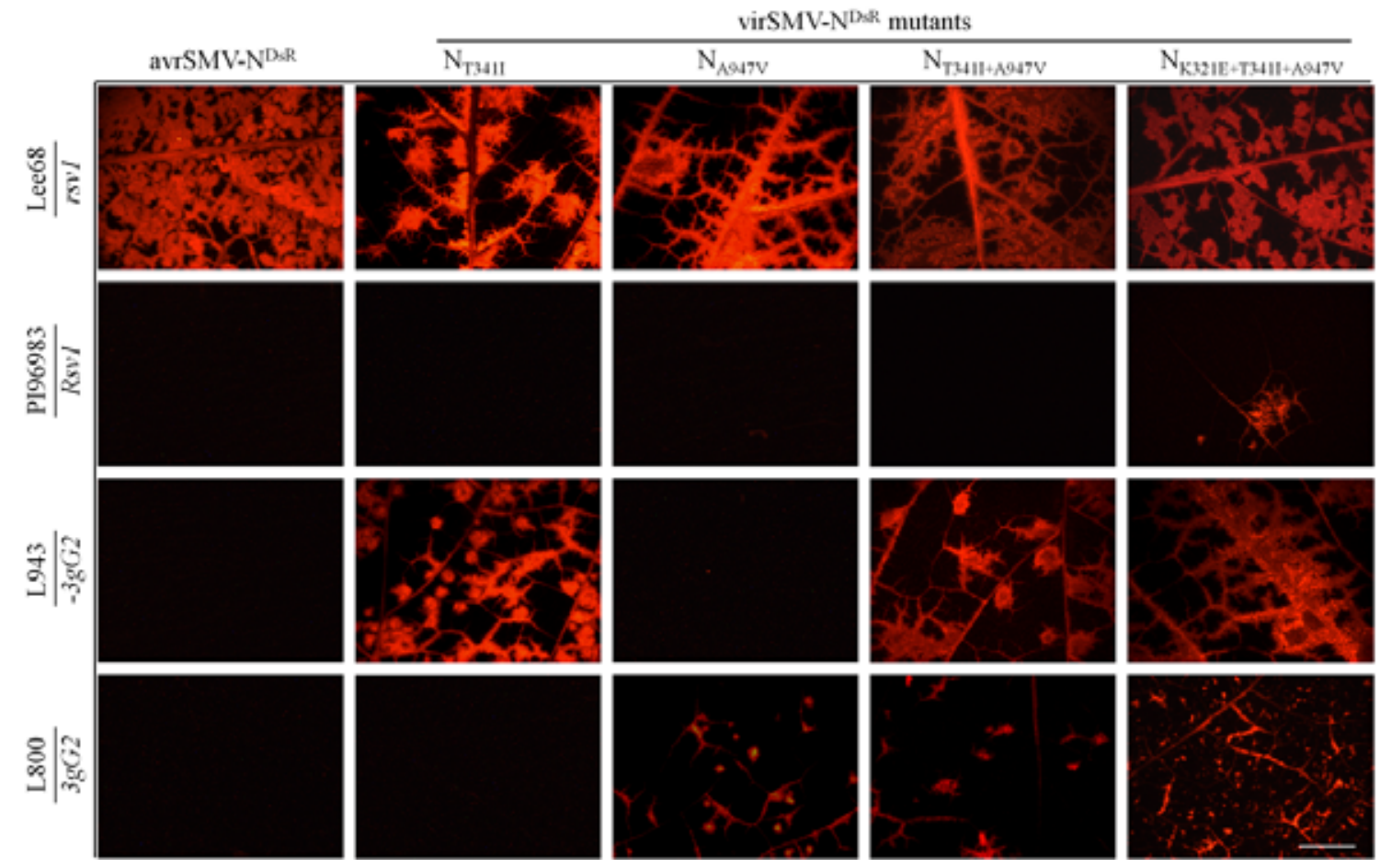

Fig. 5. Systemic infection of 'Lee68' ( $r s v 1)$, plant introduction (PI) 'PI96983' (Rsv1), and two recombinant inbred soybean lines, L943 (-3gG2) and L800 $(3 g G 2)$, with avirulent (avr)SMV-N ${ }^{\text {DsR }}$ or its derivative virulent (virSMV-N ${ }^{\text {DsR }}$ ) mutants. Attached primary leaves of 2-week-old seedlings were inoculated biolistically with molecularly cloned viruses. Inoculated plants were maintained at $22^{\circ} \mathrm{C}$ until leaflets from the trifoliate leaves were visualized and photographed under UV light for the expression of DsRed at 21 days postinoculation utilizing the same magnification of an Olympus fluorescence stereomicroscope. Scale bar $=3 \mathrm{~mm}$. Note that $\mathrm{SMV}-\mathrm{N}_{\mathrm{T} 341 \mathrm{II}}{ }^{\mathrm{Ds}}$, with a single mutation in the helper-component proteinase (HC-Pro), infected L943 ( $\left.-3 g G 2\right)$ whereas SMV-N $\mathrm{N}_{\mathrm{A} 947 \mathrm{~V}}{ }^{\mathrm{DsR}}$, with a single mutation in the P3, infected L800 evident by expression of DsRed. SMV-N $\mathrm{N}_{\mathrm{T} 341 \mathrm{I}+\mathrm{A} 947 \mathrm{~V}}$ DsR , with combined mutations in both HC-Pro and P3, infected L943 (-3gG2) and L800 (3gG2) but not PI96983 (Rsv1). SMV-N $\mathrm{K}_{221 \mathrm{I}+\mathrm{T} 341 \mathrm{I}+\mathrm{A} 947 \mathrm{~V}}$, with two mutations in the HC-Pro (K321E+T341I) combined with a single mutation in the P3 (A947V), infected all soybean genotypes. 
similar to the parental viruses virSMV-G7 and virSMV-G7d, remained virulent not only on L800 (3gG2) and L943 (-3gG2) but also on PI96983 (Rsvl) (Fig. 4). These observations suggest that it is the strain-specific $\mathrm{N}$ terminus of $\mathrm{P} 3$ that determines SMV virulence or avirulence functions on L800 (3gG2) but not on L943 $(-3 g G 2)$.

\section{Amino acid substitutions in the $P 3$ cistron of avrSMV-N alone confer virulence on L800 (3gG2) but not on L943 (-3gG2).}

Nonsynonymous mutations in the P3 cistron of avrSMV-N alone were previously shown to confer virulence on L800 (3gG2) but not on PI 96983 (Rsv1) (Hajimorad et al. 2011; Wen et al. 2011). To evaluate whether the same mutations in the P3 of avrSMV-N influences virulence of avrSMV-N on L943 (-3gG2), progeny mutant viruses in sap were mechanically inoculated onto parental soybean genotypes Lee68 ( $r s v 1)$ and PI96983 $(R s v 1)$ as well as L943 $(-3 g G 2)$ and L800 (3gG2) (Table 1). As expected, all the P3-mutants infected Lee68 ( $r s v 1)$ and none was virulent on PI96983 (Rsv1), an exception being SMV-N $\mathrm{R}_{\mathrm{R} 787+\mathrm{A} 947 \mathrm{~T}}$, where virulence was associated with a novel A350V substitution in the HC-Pro. All the P3-mutants, except SMV-N $\mathrm{N}_{\mathrm{M} 157 \mathrm{I}}$, were virulent on L800 (3gG2) without accumulation of any additional amino acid substitutions in either HC-Pro or P3 (Table 1), in agreement with previous reports (Hajimorad et al. 2011; Wen et al. 2011). Progeny viruses from none of the avrSMV-N-derived P3mutants gained virulence on L943 $(-3 g G 2)$ unless they also accumulated at least one amino acid substitution in the HC-Pro cistron (Table 1). Altogether, these observations further confirm that the genetic factors derived from the Rsv1 locus in L800 (3gG2) recognize P3 of avrSMV-N as the avirulence factor.

\section{Single amino-acid substitutions in $\mathrm{HC}$-Pro and P3 in combination convert avrSMV-N to virulence on $\mathrm{L943}$ $(-3 g G 2)$ and L800 (3gG2) but not on PI96983 (Rsv1).}

To confirm that only a single amino acid substitution in HCPro or P3 is sufficient to convert avrSMV-N to virSMV-N on L943 (-3gG2) and L800 (3gG2), respectively, we introduced the T341I and A947V mutations individually or in combination into avrSMV-N tagged with DsRedT4 (DsR) (avrSMV-N ${ }^{\text {DsR }}$ ) (Hajimorad et al. 2011). Direct biolistic inoculation of Lee68 (rsvl) showed that all the mutants replicated efficiently and moved systemically, as was evident by the expression of DsRed in the noninoculated trifoliate leaves of the inoculated plants (Fig. 5). However, SMV-N ${ }_{\mathrm{T} 341 \mathrm{I}}{ }_{\mathrm{DsR}}$ was virulent only on L943 $(-3 g G 2)$, whereas SMV-N ${ }_{\mathrm{A} 947 \mathrm{~V}}{ }^{\mathrm{DsR}}$ was virulent only on L800 $(3 g G 2)$ (Fig. 5). It should be noted that SMV-N ${ }_{\mathrm{T} 341 \mathrm{II}}{ }_{\mathrm{DsR}}$ induced systemic mosaic in $\mathrm{L} 943(-3 g G 2)$, with no apparent necrosis (data not shown), whereas SMV-N ${ }_{\mathrm{A} 947 \mathrm{~V}}{ }^{\mathrm{DSR}}$ caused systemic HR similar to the untagged virus (Wen et al. 2011). When both the T341I and A947V mutations were introduced simultaneously into the HC-Pro and the P3 cistrons of avrSMV-N ${ }^{\mathrm{DsR}}$, biolistic inoculation showed that $\mathrm{SMV}-\mathrm{N}_{\mathrm{T} 341 \mathrm{I}+\mathrm{A} 947 \mathrm{~V}}{ }^{\mathrm{DsR}}$ was virulent on both L943 ( $-3 g G 2)$ and L800 (3gG2) but not on PI96983 (Rsvl) (Fig. 5). Phenotypic responses of L943 ( $-3 g G 2)$ and L800 ( $3 g G 2)$ to inoculation with SMV-N $\mathrm{N}_{\mathrm{T} 341 \mathrm{I}+\mathrm{A} 947 \mathrm{~V}}{ }^{\mathrm{DsR}}$ were similar to those induced by individual mutants, systemic mosaic, and systemic HR, respectively (data not shown). However, introduction of the additional $\mathrm{K} 321 \mathrm{E}$ substitution in the HC-Pro of SMV-N $\mathrm{N}_{\mathrm{T} 341 \mathrm{II}+\mathrm{A} 947 \mathrm{~V} \text { DsR }}$ resulted in virulence of SMV$\mathrm{N}_{\mathrm{K} 321 \mathrm{E}+\mathrm{T} 341 \mathrm{I}+\mathrm{A} 947 \mathrm{~V}}{ }^{\mathrm{DsR}}$ on PI96983 $(R s v 1)$ as well as on L943 $(-3 g G 2)$ and L800 (3gG2) (Fig. 5; Table 1). Interestingly, SMV-N ${ }_{\mathrm{K} 321 \mathrm{E}+\mathrm{T} 341 \mathrm{I}+\mathrm{A} 947 \mathrm{~V}{ }^{\mathrm{DR}}}$ also induced systemic mosaic and systemic HR in L943 $(-3 g G 2)$ and L800 $(3 g G 2)$, respectively (data not shown). These observations confirm that HC-Pro of avrSMV-N acts as the avirulence factor on L943 $(-3 g G 2)$ whereas P3 acts as the avirulence factor on L800 (3gG2), and that virulence of avrSMV-N on PI96983 $(R s v l)$ requires at least three simultaneous mutations in HC-Pro and P3.

\section{The class G NB-LRR genes are strong candidates to encode the $R s v 1$ resistance specificities recognizing P3 or HC-Pro.}

It has been shown previously that six class G CC-NB-LRR genes are clustered at the Rsvl locus in PI96983 (Rsvl) (Hayes

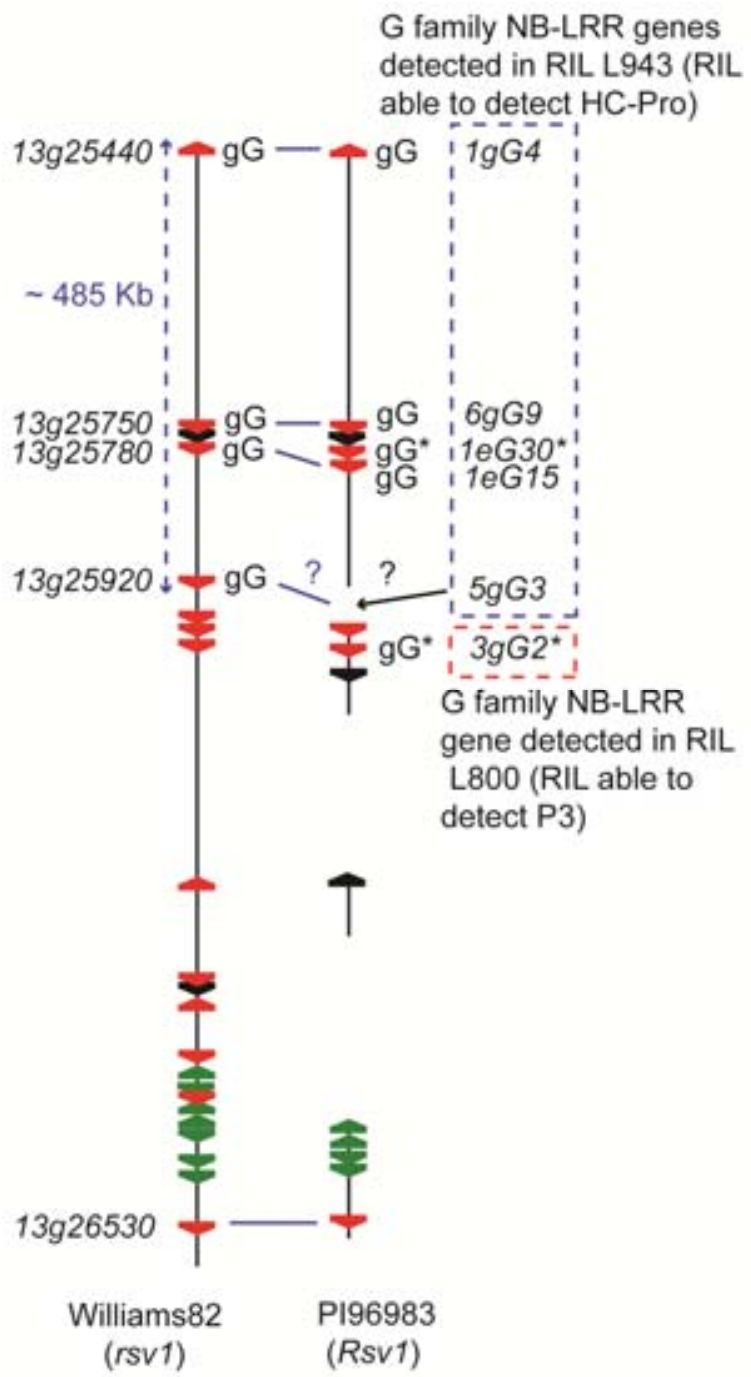

Fig. 6. Distribution of nucleotide-binding leucine-rich repeat (NB-LRR) genes across the soybean genomic region associated with the P3 and helpercomponent proteinase (HC-Pro) recognition specificities found at the Rsv1 locus. Vertical black lines represent the genomic sequence associated with the Rsv1 locus in 'Williams82' (rsv1) and plant introduction (PI) 'PI96983' $(R s v 1)$; breaks in the lines indicate regions where sequence is not available. The region shown for Williams 82 represents approximately $1 \mathrm{Mb}$. Non-toll interleukin-1 receptor (TIR) and TIR-NB-LRR genes are indicated by red and green polygons, respectively. Non-TIR-NB-LRR pseudogenes and those extending beyond the available PI96983 (Rsv1) sequence are shown in black. Glyma gene names are given to the left of the Williams82 ( $r s v 1)$ sequence. Class G NB-LRR genes (Hayes et al. 2004) are labeled with "gG", and G class genes found in recombinant inbred soybean lines L943 $(-3 g G 2)$ and L800 (3gG2) are boxed within blue and red dotted lines, respectively. Likely allelic non-TIR-NB-LRR genes in the Williams82 (rsv1) and PI96983 (Rsv1) sequences are linked by blue lines. Class G genes present in PI96983 (Rsv1) which lack apparent alleles in Williams82 ( $r s v 1)$ are marked with asterisks. The position of the $5 g G 3$ gene could not be determined unambiguously due to a gap in the available PI96983 (Rsv1) sequence but its putative location and probable allele in the Williams 82 ( $r s v 1)$ sequence are indicated with question marks. 
et al. 2004). The P3-recognizing RIL L800 contains a single class G NB-LRR gene (3gG2) from PI96983 (Rsv1) whereas RIL L943, which is able to detect the SMV HC-Pro, does not contain $3 g G 2$ but does harbor the five other family members (Hayes et al. 2004). In order to investigate the extent and complete NB-LRR gene content of the PI96983 DNA present in the vicinity of the Rsvl locus in the two RIL, we determined the distribution of the class $\mathrm{G}$ genes across genomic sequences available for the $R s v 1$ locus for the soybean lines Williams82 (rsvl) and PI96983 (Rsv1) (Innes et al. 2008).

This analysis revealed that the class $\mathrm{G}$ genes are distributed over approximately $485 \mathrm{~kb}$ in Williams82 soybean ( $r s v 1$ ) (Fig. 6). The corresponding region in PI96983 (Rsvl) is probably slightly larger due to the presence of additional NB-LRR paralogues, although a gap in the available sequence precludes an accurate estimate. It is apparent that few additional NBLRR paralogues are interspersed with the class $G$ genes (none in Williams82 [rsvl] and only one in the available PI96983 [Rsvl] genomic sequence). Furthermore, by combining the physical position data presented in Figure 6 with phylogenetic analysis of the PI96983 (Rsvl) and Williams82 (rsvl) NBLRR paralogues (Ashfield et al. 2012; data not shown), it is clear that two of the class G NB-LRR paralogues found in PI96983 (Fig. 6, marked with asterisks), including $3 g G 2$, lack alleles in Williams82 ( $r s v 1)$, consistent with them encoding resistance capabilities present in PI96983 (Rsvl) but absent in Williams82 ( $r s v 1)$.

Although additional RIL will need to be characterized to further delimit the genetic intervals containing the P3 and HC-Pro resistance specificities, this analysis indicates that the class G NB-LRR genes are strong candidates to encode one or both of the recognition specificities associated with the Rsvl locus.

\section{DISCUSSION}

The goal of this study was to understand the genetics underlying recognition of the two cistrons of avrSMV-N, HC-Pro and P3, which act as avirulence determinants in the SMVRsvl pathosystem. When compared with many other viral pathosystems involving $R$ genes, the presence of two distinct avirulence determinants within a single viral strain is uncommon. In general, $R$-gene-mediated recognition of an avirulent pathogen is based on a gene-for-gene interaction where recognition is conditioned through matched specificity between products of an $R$ gene and a pathogen avirulence gene, with recognition achieved via either direct or indirect interactions (Bonas and Lahaya 2002; Dangl and Jones 2001; Flor 1971). However, a few exceptions have been documented. For example, a rare bacterial pathosystem has been reported (Bisgrove et al. 1994; Grant et al. 1995) where two genetically unrelated avirulence genes, $a v r B$ and avrRpml from races of Pseudomonas syringae, are recognized by a single Arabidopsis gene, $R P M 1$. But, in this instance, the RPM1-mediated recognition is indirect (Mackey et al. 2002). Deviation from the classical gene-for-gene interaction has also been reported in other pathosystems. For example, the Rpgl-mediated resistance to Puccinia graminis f. sp. tritici is activated by the concerted action of two avirulence spore effectors (Nirmala et al. 2011), and avirulent strains of the bacteria Pseudomonas syringae and Ralstonia solanacearum and the fungal pathogen Colletotrichum higginsianum are perceived by two linked Arabidopsis resistance genes, RPS4 and RRS1 (Birker et al. 2009; Narusaka et al. 2009).

For plant viral pathosystems, such as those involving tobamoviruses and potexviruses, gene-for-gene type interactions have been observed (Bendahmane et al. 2000; Culver 1997). In these instances, the avirulence determinants have been mapped to different viral-encoded proteins, including the coat protein (Bendahmane et al. 2000; Gilardi et al. 2004), helicase (Erickson et al. 1999; Meshi et al. 1988), or movement protein (Malcuit et al. 1999; Meshi et al. 1989; Pfitzner and Pfitzner 1992). Unlike tobamoviruses and potexviruses, where functional proteins are expressed from a separate ORF for each protein, the entire genome of potyviruses is expressed as a single large ORF that is processed posttranslationally to produce functional proteins (Urcuqui-Inchima et al. 2001). Despite this fundamental difference in genome expression strategy, in a number of potyviral pathosystems involving $R$-gene-mediated resistance, a single cistron acts as the avirulence factor (Chowda-Reddy et al. 2011; Janzac et al. 2010; Jenner et al. 2000; Khatabi et al. 2012; Kim et al. 2010; Seo et al. 2009a; Zhang et al. 2009).

Nevertheless, there are a few potyviral pathosystems which defy the simple gene-for-gene arrangement in which a single cistron in the virus is detected by a single $\mathrm{R}$ protein in the plant. In these instances, more than one viral cistron acts as the avirulence factor. For example, the genetic determinants of Tobacco etch virus eliciting vascular necrosis in Tabasco pepper mapped to the $3^{\prime}$ part of P3 and $5^{\prime}$ part of CI, and the presence of both regions was found essential for avoiding the necrosis response (Chu et al. 1997). Similarly, the avirulence determinants of a strain of Lettuce mosaic virus provoking wilting in some resistant lettuce cultivars were mapped to P1 and CI (Krause-Sakate et al. 2005). Unlike the SMV-Rsvl pathosystem, the genetic basis of vascular necrosis in Tabasco pepper and systemic wilting in the lettuce cultivars have not been determined. However, another potyvirus, Turnip mosaic virus, requires nonsynonymous mutations in $\mathrm{P} 3$ and $\mathrm{CI}$ to gain virulence on Brassica napus line 165. However, in this instance, the involvement of two cistrons was shown to be due to the existence of two independent $R$ genes recognizing the virus (Jenner et al. 2002).

Interestingly, in a small number of pathosystems, it has been shown that resistance to a single pathogen strain requires co-expression of pairs of $R$ genes. For example, involvement of more than one NB-LRR protein against Tobacco mosaic virus in tobacco and the requirement for two toll interleukin-1 receptor (TIR)-NB-LRR genes for effective resistance against Peronospora parasitica isolate Cala2 in Arabidopsis and against Magnaporthe oryzae in rice have been reported (Ashikawa et al. 2008; Lee et al. 2009; Peart et al. 2005; Sinapidou et al. 2004). These observations have led to the suggestion that some disease-resistance pathways involve more than one NB-LRR protein (Eitas and Dangl 2010; Peart et al. 2005).

In the current study, by taking advantage of two soybean RIL with recombination events within the Rsvl locus, we demonstrated that each line recognized the product of a different cistron of avrSMV-N. P3 was recognized as the elicitor of resistance responses in RIL L800 (3gG2) whereas HC-Pro was recognized by the $R s v 1$-derived genetic factors in soybean RIL L943 (-3gG2). These observations suggest that recognition of both HC-Pro and P3 in PI96983 (Rsvl) is associated with the multigenic nature of the Rsvl locus. The nature of avrRsvl, however, is unknown, and it remains to be demonstrated whether the HC-Pro and P3 peptides are each recognized directly as a single mature functional protein. Neither is it known whether the avirulence function can be dissociated from replication in a transient gene-expression assay system, where none of the other SMV-encoded proteins are present (Abbink et al. 1998; Bendahmane et al. 2000).

The Rsvl locus is associated with a cluster of resistance specificities located on soybean chromosome 13 (formerly MLG-F) 
(Gore et al. 2002; Yu et al. 1996). This region of the soybean genome has been shown to be rich in both non-TIR and TIRclass NB-LRR genes distributed over more than a megabase of genomic DNA (Ashfield et al. 2012; Innes et al. 2008). The resistance mediated by the $R s v 1$ locus has been previously shown to be tightly linked with a subgroup of the non-TIR NB-LRR genes, referred to as class $G$ paralogues in the current study, defined using a family-specific hybridization probe (Hayes et al. 2004). In the study by Hayes and associates (2004), it was determined that the L800 (3gG2) RIL contains only one of the G-class genes from the resistant PI96983 (Rsvl) parent. In contrast, the L943 (-3gG2) RIL does not share $3 g G 2$ with L800 but, rather, contains five other genes from this subfamily (IeG30, $5 g G 3$, IeG15, $6 g G 9$, and $I g G 4)$. Despite the tight genetic linkage, it is unknown which, if any, of these class $\mathrm{G}$ genes mediate recognition of the SMV strainspecific HC-Pro. However, the analyses presented here are consistent with G-class paralogues encoding one or both of the resistance specificities. By locating the class $G$ paralogues in the genomic sequences from both the Rsv1-expressing soybean PI96983 and from a cultivar that lacks this resistance specificity (Williams82), we showed that only a few other NB-LRR genes are interspersed with this subfamily and would be expected to co-segregate with resistance to SMV. Furthermore, we observed that at least two of the class $G$ genes found in PI96983 (Rsv1) lack alleles in Williams82 ( $r s v 1)$, suggestive of a role in a resistance phenotype found in PI96983 (Rsv1) but not in Williams82 ( $r s v 1)$. However, a role for other subfamilies of NB-LRR genes in the Rsv1 phenotype cannot be excluded and additional genetic and functional analyses will be required to identify the specific paralogues responsible for recognition of P3 and HC-Pro. Interestingly, in a phylogenetic analysis of the approximately 390 NB-LRR genes found in Williams82 $(r s v 1)$, the non-TIR NB-LRR paralogues (including the class G genes) found in the immediate vicinity of the Rsvl locus are clustered in two well-supported sister clades (Zhang et al. 2011). This observation is consistent with the P3- and HC-Prospecific $R$ genes sharing a relatively close evolutionary relationship and raises the possibility that some attribute of the NB-LRR genes in this region predisposes them for a role in triggering resistance to SMV.

RIL L800 (3gG2) expresses ER against avrSMV-N, similar to PI96983 (Rsv1), whereas L943 ( $-3 g G 2)$ allows for limited replication at the inoculation site. These observations indicate that $3 g G 2$ or another tightly linked gene in L800 is sufficient for $R s v 1$-mediated ER expression against avrSMV-N. Furthermore, they suggest that the mechanism of resistance in L943 $(-3 g G 2)$ against avrSMV-N may differ from that of L800 $(3 g G 2)$ and appears to target virus movement; however, an alternative possibility is that the resistance displayed by L943 $(-3 g G 2)$ simply represents a weaker triggering of the same pathways that are activated in L800 (3gG2). It should be noted that L943 $(-3 g G 2)$ expresses mild mosaic in interactions with virSMV-G7 whereas L800 (3gG2) expresses systemic HR. Furthermore, overcoming resistance operating in L800 (3gG2) by avrSMV-N-derived P3 mutants also leads to induction of systemic HR (Hajimorad et al. 2011; Wen et al. 2011). Interestingly, a few of the avrSMV-N-derived HC-Pro mutants also provoked systemic HR in L943 $(-3 g G 2)$, despite the fact that the majority induced systemic mosaic with no apparent necrosis (data not shown). These observations suggest that, if suitably provoked, both L800 $(3 g G 2)$ and L943 $(-3 g G 2)$ are capable of activating a similar $R s v 1$-associated defense pathway.

In soybean, in addition to Rsvl, two other loci, Rsv3 and $R s v 4$, also confer resistance to SMV. The genomic regions encompassing these loci have been previously characterized in the susceptible Williams82 (Saghai Maroof et al. 2010; Suh et al. 2011) and their gene content may help to explain the similarities or differences in the resistance phenotypes conferred by the $R s v$ loci. Like Rsv1, Rsv3 is also associated with a cluster of non-TIR NB-LRR genes (Suh et al. 2011) although, in this case, it is the CI protein that is recognized (Seo et al. 2009a; Zhang et al. 2009). Furthermore, both Rsv1 and Rsv3 loci invoke ER or HR to avirulent SMV strains, if provoked. Together, these observations suggest that, despite recognizing different avirulence determinants in SMV, the P3-specific gene at the Rsvl locus and the CI-specific gene at the Rsv3 locus may be triggering related resistance pathways. Intriguingly, a greater number of alleles have been identified (Moon et al. 2009) for the Rsvl locus than for $R s v 3$ (nine for $R s v 1$ versus three for $R s v 3$ ), perhaps reflecting the potential for creation of different resistance specificities due to rearrangements at the $R s v l$ locus. Interestingly, the $R s v 4$ locus has very different attributes and appears not to be associated with an NB-LRR cluster (Saghai Maroof et al. 2010). Unlike the $R s v 1$ and $R s v 3$ loci, the resistance conferred by the Rsv4 locus is not associated with HR or ER but, instead, is characterized by delayed virus replication and movement (Gunduz et al. 2004). The genomic region encompassing the Rsv4 locus has been characterized in the rsv4 line Williams 82 and, although no NBLRR genes are present, several transcription factor genes (MADS or MYB families) were identified (Saghai Maroof et al. 2010). These observations are consistent with Rsv4 utilizing a different mode of resistance that is not dependent on NB-LRRmediated recognition.

The observation that an avrSMV-N-derived mutant with a single nonsynonymous mutation in each of the HC-Pro and P3 cistrons was virulent on both L943 ( $-3 g G 2)$ and L800 $(3 g G 2)$ but not PI96983 (Rsvl) is interesting. To functionally convert avrSMV-N to virSMV-N on PI96983 (Rsv1), at least one additional nonsynonymous mutation is required in the HC-Pro or P3 cistrons (Eggenberger et al. 2008; Hajimorad et al. 2011). PI96983 (Rsv1), unlike L943 (-3gG2) and L800 (3gG2), contains the entire complex chromosomal region of the Rsvl locus, and this raises the possibility that either host genetic background may have an impact or that PI96983 (Rsvl) has an additional resistance gene recognizing avrSMV-N that is absent in both L943 (-3gG2) and L800 (3gG2). It has been shown in other pathosystems that, in addition to $R$ and $A v r$ gene products, additional host factors are also required for $R$-gene-mediated signaling (Banerjee et al. 2001; Jones and Dangl 2006). The observation that K321E substitution in HC-Pro is essential for virulence of SMV-N $\mathrm{K}_{\mathrm{K} 321 \mathrm{E}+\mathrm{T} 341 \mathrm{I}+\mathrm{A} 947 \mathrm{v}}$ on PI96983 (Rsv1) supports this possibility. It should be noted that SMV-N $\mathrm{K}_{\mathrm{K} 321 \mathrm{E}}$ remained avirulent on both L943 ( $-3 g G 2)$ and L800 (3gG2), unless other, nonsynonymous mutations were selected in either the HC-Pro or P3 cistrons. We have also noticed previously that, for gain of virulence of avrSMV-N on L78-379 (Rsv1), an isoline of Williams containing the Rsvl locus from PI96983 (Bernard et al. 1991), additional mutations in HC-Pro or P3 are required when compared with gain of virulence by the same virus on PI96983 (Rsv1) (Hajimorad et al. 2008, 2011).

The involvement of more than one resistance gene derived from the Rsvl locus targeting two cistrons of SMV for recognition offers a plausible explanation for lack of widespread, naturally occurring virulent strains. To date, naturally occurring virulent strains of SMV on Rsv3 and Rsv4 have been reported in North America and South Korea (Chowda-Reddy et al. 2011; Gunduz et al. 2004; Khatabi et al. 2012; Seo et al. 2009b; Viel et al. 2009). In contrast, excluding virSMV-G7, naturally occurring virulent strains on Rsvl have been documented only in South Korea (Choi et al. 2005). CI and P3 of SMV act as avrRsv3 and avrRsv4 cistrons, respectively, and it has been shown that nonsynonymous mutations in CI or P3 cistrons can functionally convert avirulence to virulence on 
$R s v 3$ and Rsv4-genotype soybean, respectively (ChowdaReddy et al. 2011; Khatabi et al. 2012; Seo et al. 2009a; Zhang et al. 2009). In contrast, as shown previously and in this article, for functional conversion of avrSMV-N to virSMV-N on $R s v 1$-genotype soybean, not only are mutations in two cistrons essential but also at least three simultaneous amino acid substitutions in HC-Pro and P3 are required (Eggenberger et al. 2008; Hajimorad et al. 2008, 2011). The required substitutions could be at least one amino acid in HC-Pro and two in P3 (Eggenberger et al. 2008) or two in HC-Pro and one in P3 (Hajimorad et al. 2011). Durability of $R$ genes against plant viruses has been attributed partly to a requirement for a high number of simultaneous mutations in the corresponding viral pathogens to achieve virulence (Harrison 2002). Other predictors of durability include evolutionary constraints acting on avirulence factors for amino acid substitution and high fitness cost associated with gain of virulence (Janzac et al. 2009, 2010). In the case of SMV, P3 is under lesser evolutionary constraints compared with the other cistrons (Seo et al. 2009b).

It should be noted that virulent variants derived from molecularly cloned avrSMV-N were relatively easily selected on L800 (3gG2) via experimental adaptation (Hajimorad et al. 2011). However, despite many attempts, we have failed to select such a virulent variant directly on PI96983 (Rsv1). This failure is possibly due to the complementary functions of the $\mathrm{P} 3$-specific $R$ gene present in L800 and the HC-Pro-specific gene in L943 (and possibly an additional third gene at the Rsvl locus). Enhancement of resistance durability due to complementary functions of more than one resistance gene has been reported in another potyviral pathosystem (Acosta-Leal and Xiong 2008). Thus, these observations collectively suggest that $R s v 1$ mediated resistance will be relatively durable under field conditions. Furthermore, our data suggest that, to exploit such durable resistance in breeding programs, it will be necessary to introduce the entire $R s v 1$ locus region and not just one of the component $R$ genes. Identification of the specific NB-LRR paralogues responsible for recognition of HC-Pro and P3 will likely help define the minimum region required for durable resistance and thus facilitate breeding efforts.

\section{MATERIALS AND METHODS}

\section{Viruses, soybean genotypes, inoculation, and SMV detection.}

The previously described infectious cDNA clones of SMV-N (pSMV-N) (accession number D00507), SMV-G7 (pSMV-G7) (accession number AY216010), and SMV-G7d (pSMV-G7d) (accession number AY216987) served as the sources of parental viruses. The modified genome of SMV-N to serve as a gene vector in plants (pSMV-Nv) as well as pSMV-N-GUS and pSMV-N ${ }^{\mathrm{DsR}}$ have been described previously (Hajimorad et al. 2011; Wang et al. 2006). All plasmids were propagated in ElectroMax DH5 $\alpha-E$ (Invitrogen, Carlsbad, CA, U.S.A.) and purified by using a QiaPrep spin miniprep kit (Qiagen, Valencia, CA, U.S.A.).

To establish infection with plasmid DNA, fully expanded primary leaves of soybean seedlings were biolistically inoculated as described previously (Hajimorad et al. 2003, 2008). Sap containing viral progenies in $50 \mathrm{mM}$ phosphate buffer, $\mathrm{pH}$ 7.0, derived from the infected tissues of biolistically inoculated Williams82 ( $r s v 1)$ served as inoculum to mechanically inoculate Carborundum (600-mesh)-dusted primary leaves of other soybean genotypes. Soybean (Glycine max) genotypes Williams82 (rsv1) and Lee68 (rsv1), both universally susceptible to all strains of SMV, and Rsv1-genotype soybean PI96983, RIL L800 (3gG2), and RIL L943 (-3gG2) were used (Bernard et al. 1991; Hayes et al. 2004). PI96983 (Rsvl) is a soybean PI containing the Rsvl resistance locus region that was originally collected in Hwanghae Puk, South Korea, and introduced to the United States in 1932. Soybean L800 (3gG2) and L943 $(-3 g G 2)$ were selected from a population resulting from the crossing of Lee68 ( $r s v l)$ with PI96983 (Rsvl) (Hayes et al. 2004). Soybean L800 (3gG2) is an RIL containing only one of the six G family NB-LRR candidate resistance genes $(3 g G 2)$ at the Rsvl chromosomal region from PI96983 (Rsvl) (Hayes et al. 2004). In contrast, soybean L943 is another RIL which lacks $3 g G 2$ but, instead, contains five other class $\mathrm{G}$ genes at the Rsv1 chromosomal region from PI96983 (Rsvl) (Hayes et al. 2004). All soybean seed were obtained from field-grown plants shown to be free of SMV by indexing. The inoculated plants were maintained in a growth chamber operating at $22^{\circ} \mathrm{C}$ with a photoperiod of $16 \mathrm{~h}$. Detection of SMV in the inoculated plants was done by symptomatology, enzyme-linked immunosorbent assay, reverse-transcriptase polymerase chain reaction (RT-PCR), histochemical assay of GUS expression, or visualization of DsRed expression with an Olympus fluorescence stereomicroscope equipped with a filter set for excitation at 530 to $560 \mathrm{~nm}$ and an emission at 590 to $650 \mathrm{~nm}$ (Hajimorad et al. 2011; Malapi-Nelson et al. 2009; Wen and Hajimorad 2010).

\section{Synthesis of HC-Pro-derived chimeras with precise replacement of $\mathrm{HC}$-Pro.}

To construct the chimeras with precise exchanges of HC-Pro among avrSMV-N, virSMV-G7, and virSMV-G7d, fusionPCR, as described by Charlier and associates (2003), was used. The oligonucleotide primers used are listed in Supplementary Table S2. PrimeSTAR HS DNA polymerase (Takara Bio, Madison, WI, U.S.A.) was used for the construction of all the chimeras.

To generate pSMV-N/G7HC-Pro (SMV-N/G7HC-Pro) or pSMV-N/G7dHC-Pro (SMV-N/G7dHC-Pro), initially, the primer sets Nots/P1a and G7P3s/N-3319a were used to amplify two fragments consisting of sequences located upstream or downstream of SMV-N HC-Pro independently while pSMV-N served as the template. The primer pairs HCs and G7-HCa was used in PCR to amplify the full-length sequences of HC-Pro of virSMV-G7 or virSMV-G7d from pSMV-G7 or pSMV-G7d templates. For fusion-PCR, the above three gel-purified, PCRamplified fragments were mixed together at the same molar ratio to serve as the template in a $50-\mu l$ reaction mixture that consisted of $800 \mathrm{ng}$ of mixed fragments, $5 \mathrm{U}$ of PrimeSTAR HS DNA polymerase, $10 \mu \mathrm{l}$ of $5 \times$ buffer supplied by the manufacturer, $500 \mu \mathrm{M}$ mixture of dNTPs, and $0.5 \mu \mathrm{M}$ each of primers Nots and N-3319a. The PCR program for the amplification reaction was as follows: a cycle of $3 \mathrm{~min}$ at $95^{\circ} \mathrm{C} ; 10 \mathrm{~s}$ at $98^{\circ} \mathrm{C}, 30 \mathrm{~s}$ at $60^{\circ} \mathrm{C}$, and $4 \mathrm{~min}$ at $72^{\circ} \mathrm{C}$ for 30 cycles; followed by an additional extension step at $72^{\circ} \mathrm{C}$ for $10 \mathrm{~min}$. The resultant amplified fragments were digested with NotI and SpeI and ligated into $\mathrm{pSMV}-\mathrm{N}$ to generate $\mathrm{pSMV}-\mathrm{N} / \mathrm{G} 7 \mathrm{HC}$-Pro or pSMV-N/G7dHC-Pro.

To generate pSMV-G7/NHC-Pro (SMV-G7/NHCPro) or pSMV-G7d/NHC-Pro (SMV-G7d/NHC-Pro), primer sets Nots/ P1a and N-P3s/G7-3285a were used to amplify two fragments consisting of sequences upstream and downstream of HC-Pro independently using pSMV-G7 or pSMV-G7d as templates. The primer set of $\mathrm{HCs}$ and $\mathrm{N}-\mathrm{HCa}$ was used subsequently to amplify the full-length HC-Pro of avrSMV-N while using pSMV-N as the template. For the fusion-PCR, the above three PCR-amplified fragments were gel purified and mixed together with a similar molar ratio and used as the template in a $50-\mu 1$ reaction mixture as described above, except Nots and G7-3285a served as primers. The resultant amplicons were digested with NotI and SpeI and ligated into similarly digested pSMV-G7 or 
pSMV-G7d to generate pSMV-G7/NHC-Pro or pSMV-G7d/ NHC-Pro.

To ensure absence of any undesired PCR-generated mutation, the entire PCR-amplified regions of each of the chimeric viruses were sequenced and analyzed. The infectivity of the resultant chimeras was tested by biolistic inoculation on Williams82 (rsv1) (Hajimorad et al. 2008).

\section{SMV chimeras with exchanged $P 3$ sequences.}

Chimeric viruses SMV-N/G7P3, SMV-N/G7dP3, SMVG7/NP3, and SMV-G7d/NP3 that contain precise exchanges of P3 have previously been described (Hajimorad et al. 2006). Other P3-derived chimeras with only the $\mathrm{N}$ - or C-termini exchanges among avrSMV-N, virSMV-G7, and virSMV-G7d also have been described (Hajimorad et al. 2006). Progeny viruses derived from these chimeras that served as inoculum in-

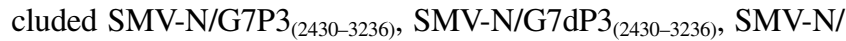
G7P3 $3_{(3237-3626)}$, SMV-N/G7dP3 $3_{(3237-3626)}$, SMV-G7/NP3 $3_{(2427-3233)}$, SMV-G7d/NP3 $3_{(2427-3233)}$, SMV-G7/NP3 $3_{(3234-3623)}$, and SMV-G7d/ NP3 $3_{(3234-3623)}$.

\section{SMV-N-derived HC-Pro and P3 site-directed mutants.}

SMV-N-derived mutants with one or more nonsynonymous mutations in HC-Pro cistron have previously been described (Eggenberger et al. 2008; Hajimorad et al. 2008, 2011). Progeny viruses derived from biolistically inoculated Williams82 ( $r s v 1)$ with each of the mutants served as inoculum for mechanical inoculations. The mutant viruses included $\mathrm{SMV}-\mathrm{N}_{\mathrm{F} 316 \mathrm{~S}}$, SMV-N ${ }_{\mathrm{F} 317 \mathrm{~S}}, \mathrm{SMV}-\mathrm{N}_{\mathrm{R} 318 \mathrm{P}}, \mathrm{SMV}-\mathrm{N}_{\mathrm{G} 319 \mathrm{D}}, \mathrm{SMV}-\mathrm{N}_{\mathrm{G} 319 \mathrm{~S}}, \mathrm{SMV}-$ $\mathrm{N}_{\mathrm{W} 320 \mathrm{~L}}, \mathrm{SMV}-\mathrm{N}_{\mathrm{K} 321 \mathrm{E}}, \mathrm{SMV}-\mathrm{N}_{\mathrm{G} 319 \mathrm{~S}+\mathrm{K} 321 \mathrm{E}}, \mathrm{SMV}-\mathrm{N}_{\mathrm{K} 326 \mathrm{E}}$, SMV$\mathrm{N}_{\mathrm{T} 341 \mathrm{I}}, \quad \mathrm{SMV}-\mathrm{N}_{\mathrm{K} 321 \mathrm{E}+\mathrm{T} 341 \mathrm{I}}, \mathrm{SMV}-\mathrm{N}_{\mathrm{V} 359 \mathrm{~F}}, \mathrm{SMV-N} \mathrm{C}_{\mathrm{C} 67 \mathrm{Y}}, \mathrm{SMV}-$ $\mathrm{N}_{\mathrm{R} 682 \mathrm{~K}}$, SMV-N $\mathrm{N}_{\mathrm{R} 682 \mathrm{G}}$, and SMV-N $\mathrm{R}_{\mathrm{R} 682 \mathrm{M}}$. SMV-N-derived P3 mutants containing one or more nonsynonymous mutations have previously been described (Eggenberger et al. 2008; Hajimorad et al. 2006, 2011). Progeny viruses derived from biolistically inoculated Williams82 ( $r s v 1)$ with each of the mutants served as inoculum for mechanical inoculations. The mutant viruses included SMV-N ${ }_{\mathrm{R} 787 \mathrm{I}}, \mathrm{SMV}-\mathrm{N}_{\mathrm{V} 822 \mathrm{M}}, \mathrm{SMV}-\mathrm{N}_{\mathrm{R} 945 \mathrm{G}}, \mathrm{SMV}-\mathrm{N}_{\mathrm{A} 947 \mathrm{~T}}$, SMV-N ${ }_{\mathrm{A} 947 \mathrm{~V}}, \mathrm{SMV}-\mathrm{N}_{\mathrm{P} 948 \mathrm{~L}}, \mathrm{SMV}-\mathrm{N}_{\mathrm{K} 952 \mathrm{E}}, \mathrm{SMV}-\mathrm{N}_{\mathrm{V} 1045 \mathrm{~A}}, \mathrm{SMV-}$ $\mathrm{N}_{\mathrm{M} 1157 \mathrm{I}}, \mathrm{SMV}-\mathrm{N}_{\mathrm{A} 1111 \mathrm{~V}}, \mathrm{SMV}-\mathrm{N}_{\mathrm{R} 787 \mathrm{I}+\mathrm{A} 947 \mathrm{~T}}, \mathrm{SMV}-\mathrm{N}_{\mathrm{V} 822 \mathrm{M}+\mathrm{K} 952 \mathrm{E}}$, $\mathrm{SMV}-\mathrm{N}_{\mathrm{V} 822 \mathrm{M}+\mathrm{K} 952 \mathrm{E}+\mathrm{A} 1111 \mathrm{~V}}$, and $\mathrm{SMV}-\mathrm{N}_{\mathrm{V} 822 \mathrm{M}+\mathrm{K} 952 \mathrm{E}+\mathrm{A} 1111 \mathrm{~V}+\mathrm{E} 767 \mathrm{G}}$.

\section{SMV-N-derived mutants with combined mutations in both HC-Pro and P3.}

Synthesis of a number of avrSMV-N-derived mutants with nonsynonymous mutations in both HC-Pro and P3 cistrons have previously been described (Hajimorad et al. 2011). Progeny viruses derived from biolistically inoculated Williams 82 ( $r s v 1)$ with each of these mutants served as inoculum for mechanical inoculations. These mutant viruses included SMV$\mathrm{N}_{\mathrm{K} 321 \mathrm{E}+\mathrm{A} 947 \mathrm{~V}}, \mathrm{SMV}-\mathrm{N}_{\mathrm{K} 321 \mathrm{E}+\mathrm{R} 945 \mathrm{G}}, \mathrm{SMV}-\mathrm{N}_{\mathrm{K} 321 \mathrm{E}+\mathrm{T} 341 \mathrm{I}+\mathrm{A} 947 \mathrm{~V}}$, SMV$\mathrm{N}_{\mathrm{G} 319 \mathrm{~S}+\mathrm{K} 321 \mathrm{E}+\mathrm{A} 947 \mathrm{~V}}$, and SMV-N $\mathrm{N}_{\mathrm{R} 682 \mathrm{M}+\mathrm{R} 787 \mathrm{I}+\mathrm{A} 947 \mathrm{~T}}$.

\section{Construction of DsRed-tagged viruses.}

To synthesize SMV-N $\mathrm{T}_{\mathrm{T} 341 \mathrm{I}}$ tagged with DsR, the T341I mutation was introduced into the HC-Pro cistron of pSMV-Nv as previously described (Hajimorad et al. 2008, 2011), except pSMV-Nv was used as the template. Subsequently, DsR was released by AvrII digestion of pSMV-N ${ }^{\mathrm{DsR}}$ (Hajimorad et al. 2011) and ligated into $\mathrm{pSMV}-\mathrm{Nv}_{\mathrm{T} 341 \mathrm{I}}$ to generate $\mathrm{pSMV}$ $\mathrm{N}_{\mathrm{T} 341 \mathrm{I}}{ }^{\mathrm{DsR}}$. A KpnI/SpeI fragment encoding A947V mutation was released from pSMV-N ${ }_{\mathrm{A} 947 \mathrm{~V}}$ (Hajimorad et al. 2011) and ligated into pSMV-N ${ }^{\mathrm{DsR}}$ and $\mathrm{pSMV}-\mathrm{Nv}_{\mathrm{T} 341 \mathrm{I}}$ to generate $\mathrm{pSMV}$ $\mathrm{N}_{\mathrm{A} 947 \mathrm{~V}}{ }^{\mathrm{DsR}}$ and $\mathrm{pSMV}-\mathrm{Nv}_{\mathrm{T} 341 \mathrm{I}+\mathrm{A} 947 \mathrm{~V} \text {. Finally, DsR was ligated }}$ into pSMV-N $\mathrm{N}_{\mathrm{vT} 341 \mathrm{I}+\mathrm{A} 947 \mathrm{~V}}$ to generate $\mathrm{pSMV} \mathrm{N}_{\mathrm{T} 341 \mathrm{I}+\mathrm{A} 947 \mathrm{~V}}{ }^{\mathrm{DsR}}$. pSMV-N ${ }_{\mathrm{K} 321 \mathrm{E}+\mathrm{T} 341 \mathrm{I}+\mathrm{A} 947 \mathrm{~V}}{ }^{\mathrm{DsR}}$ has previously been described (Hajimorad et al. 2011).
RNA extraction, RT-PCR, and sequencing.

Total RNA was isolated from systemically infected trifoliate leaves using an RNeasy plant mini kit (Qiagen). To verify the stability of the chimeras or site-directed mutants in planta, the genomic region of progeny viruses containing the HC-Pro and P3 cistrons was reverse transcribed using primer SMV-3910a in the presence of SuperScript reverse-transcriptase III (Invitrogen) as instructed by the manufacturer. Nested-PCR amplification of the entire HC-Pro and P3 citrons was done using two pairs of primers, SMV-214s/SMV-3910a and SMV456s/SMV-3840a, in the presence of ExTaq polymerase (Takara Bio). The resultant amplicons were purified with a QIAquick-PCR Purification Kit (Qiagen) and sequenced using primers SMV-1439s, SMV-1614a, SMV-2289s, and SMV2919s. Sequencing was done at The University of Tennessee DNA Sequencing Facility and sequences were edited and analyzed using Vector NTI (Invitrogen).

\section{Analysis of the soybean genomic region associated with the Rsv1 locus.}

The sequencing of the Rsvl genomic region from soybean Williams82 (complete sequence available) and PI96983 (partial sequence available), and the identification of NB-LRR genes within these sequences, has been reported previously (Innes et al. 2008; Schmutz et al. 2010). To identify which of these NBLRR paralogues represent the class $G$ genes described by Hayes and associates (2004), two strategies were followed. First, the three fully sequenced class $\mathrm{G}$ genes $3 g G 2,5 g G 3$, and $6 g G 9$ were compared directly with the known NB-LRR genes within the PI96983 genomic sequence using BLASTn. Second, to identify the position of the remaining class $G$ genes in the Williams82 (rsvl) and PI96983 (Rsvl) genomic sequences, advantage was taken of the sequence conservation found immediately downstream of the class $\mathrm{G}$ gene ORF (Hayes et al. $2004)$. The $1.5 \mathrm{~kb}$ of sequence immediately $3^{\prime}$ of the $3 g G 2$ ORF was used to search the genomic assemblies for related sequences using BLASTn, and regions sharing $>85 \%$ nucleotide identity, immediately downstream of predicted NB-LRR ORF, were used to identify these adjacent paralogues, as from the class $G$ subfamily. The genetically defined order of class $G$ genes across the region (Hayes et al. 2004) was then used to infer the identity of specific paralogues in the PI96983 genomic sequence. Likely allelic relationships between pairs of class G NB-LRR genes in the Williams82 and PI96983 genomic sequences were inferred when pairs of genes shared syntenic positions and had the expected relationships in phylogenetic analyses (Ashfield et al. 2012; data not shown).

\section{ACKNOWLEDGMENTS}

This project was supported, in part, by The University of Tennessee College of Agricultural Sciences and Natural Resources; the Tennessee Agricultural Experimental Station, Knoxville; North Central Soybean Research Program; United Soybean Board; Tennessee Soybean Promotion Board; and the Virginia Soybean Board.

\section{LITERATURE CITED}

Abbink, T. E. M., Tjernberg, P. A., Bol, J. F., and Linthorst, H. J. M. 1998 Tobacco mosaic virus helicase domain induces necrosis in $N$ gene-carrying tobacco in the absence of virus replication. Mol. Plant-Microbe Interact. 11:1242-1246.

Acosta-Leal, R., and Xiong, Z. 2008. Complementary functions of two recessive R-genes determine resistance durability of tobacco 'Virgin A Mutant' (VAM) to Potato virus Y. Virology 379:275-283.

Adams, M. J., Antoniw, J. F., and Fauquet, C. M. 2005. Molecular criteria for genus and species discrimination within the family Potyviridae. Arch. Virol. 150:459-479.

Ashfield, T., Egan, A. N., Pfeil, B. E., Chen, N. W. G., Podicheti, R., 
Ratnaparkhe, M. B., Ameline-Torregrosa, C., Denny, R., Cannon, S., Doyle, J. J., Geffroy, V., Roe, B. A., Saghai Maroof, M. A., Young, N. D., and Innes, R. W. 2012. Evolution of a complex disease resistance gene cluster in diploid Phaseolus and tetraploid Glycine. Plant Physiol. 159:336-354.

Ashikawa, I., Hayashi, N., Yamane, H., Kanamori, H., Wu, J., Matsumoto, T., Ono, K., and Yano, M. 2008. Two adjacent nucleotide-binding siteleucine-rich repeat class genes are required to confer $P i \mathrm{~km}$-specific rice blast resistance. Genetics 180:2267-2276.

Banerjee, D., Zhang, X., and Bent, A. F. 2001. The leucine-rich repeat domain can determine effective interaction between RPS2 and other host factors in Arabidopsis RPS2-mediated disease resistance. Genetics 158:439-450.

Bendahmane, A., Kanyuka, K., and Baulcombe, D. C. 1999. The $R x$ gene from potato controls separate virus resistance and cell death responses. Plant Cell 11:781-791.

Bendahmane, A., Querci, M., Kanyuka, K., and Baulcombe, D. C. 2000. Agrobacterium transient expression system as a tool for the isolation of disease resistance genes: Application to the $R x 2$ locus in potato. Plant $\mathrm{J}$. 21:73-81.

Bernard, R. L., Nelson, R. L., and Creemens, C. R. 1991. USDA soybean genetic collection: Isoline collection. Soybean Genet. Newsl. 18:27-57.

Birker, D., Heidrich, K., Takahara, H., Narusaka, M., Deslandes, L., Narusaka, Y., Reymond, M., Parker, J. E., and O'Connell, R. 2009. A locus conferring resistance to Colletotrichum higginsianum is shared by four geographically distinct Arabidopsis accessions. Plant J. 60:602-613.

Bisgrove, S. R., Simonich, M. T., Smith, N. M., Sattler, A., and Innes, R. W. 1994. A disease resistance gene in Arabidopsis with specificity for two different pathogen avirulence genes. Plant Cell 6:927-933.

Bonas, U., and Lahaye, T. 2002. Plant disease resistance triggered by pathogen-derived molecules: Refined models of specific recognition. Curr. Opin. Microbiol. 5:44-50.

Charlier, N., Molenkamp, R., Leyssen, P., Vandamme, A.-M., De Clercq, E., Bredenbeek, P., and Neyts, J. 2003. A rapid and convenient variant of fusion-PCR to construct chimeric flaviviruses. J. Virol. Methods 108:67-74.

Cho, E.-K., and Goodman, R. M. 1979. Strains of soybean mosaic virus: Classification based on virulence in resistant soybean cultivars. Phytopathology 69:467-470.

Choi, B. K., Koo, J. M., Ahn, H. J., Yum, H. J., Choi, C. W., Ryu, K. H., Chen, P., and Tolin, S. A. 2005. Emergence of Rsv-resistance breaking Soybean mosaic virus isolates from Korean soybean cultivars. Virus Res. 112:42-51.

Chowda-Reddy, R. V., Sun, H., Chen H., Poysa, V., Ling, H., Gijzen, M., and Wang, A. 2011. Mutations in the P3 protein of Soybean mosaic virus G2 isolates determine virulence on Rsv4-genotype soybean. Mol. Plant-Microbe Interact. 24:37-43.

Chu, M., Lopez-Moya, J. J., Llave-Correas, C., and Pirone, T. P. 1997. Two separate regions in the genome of the tobacco etch virus contain determinants of the wilting response of Tabasco pepper. Mol. PlantMicrobe Interact. 10:472-480.

Chung, B. Y.-W., Miller, W. A., Atkins, J. F., and Firth, A. E. 2008. An overlapping essential gene in the Potyviridae. Proc. Natl. Acad. Sci. U.S.A. 105:5897-5902.

Culver, J. N. 1997. Viral avirulence genes. Pages 196-219 in: PlantMicrobe Interactions, Vol. 2. G. Stacey and N. T. Keen, eds. Chapman \& Hall, New York.

Culver, J. N., Lindbeck, A. G. C., and Dawson, W. O. 1991. Virus-host interactions: Induction of chlorotic and necrotic responses in plants by tobamoviruses. Annu. Rev. Phytopathol. 29:193-217.

Dangl, J. L., and Jones, J. D. G. 2001. Plant pathogens and integrated defence responses to infection. Nature 411:826-833.

Dinesh-Kumar, S. P., Tham, W.-H., and Baker, B. J. 2000. Structure-function analysis of the tobacco mosaic virus resistance gene $N$. Proc. Natl. Acad. Sci. U.S.A. 97:14789-14794

Eggenberger, A. L., Hajimorad, M. R., and Hill, J. H. 2008. Gain of virulence on Rsv1-genotype soybean by an avirulent Soybean mosaic virus requires concurrent mutations in both P3 and HC-Pro. Mol. PlantMicrobe Interact. 21:931-936.

Eitas, T. K., and Dangl, J. L. 2010. NB-LRR proteins: Pairs, pieces, perception, partners, and pathways. Curr. Opin. Plant Biol. 13:472-477.

Erickson, F. L., Holzberg, S., Calderon-Urrea, A., Handley, V., Axtell, M., Corr, C., and Baker, B. 1999. The helicase domain of the TMV replicase proteins induces the $\mathrm{N}$-mediated defence response in tobacco. Plant J. 18:67-75.

Flor, H. H. 1971. Current status of the gene-for-gene concept. Annu. Rev. Phytopathol. 9:275-296.

Gilardi, P., García-Luque, I., and Serra, M. T. 2004. The coat protein of tobamovirus acts as elicitor of both $L^{2}$ and $L^{4}$ gene-mediated resistance in Capsicum. J. Gen. Virol. 85:2077-2085.
Gore, M. A., Hayes, A. J., Jeong, S. C., Yue, Y. G., Buss, G. R., and Saghai Maroof, M. A. 2002. Mapping tightly linked genes controlling potyvirus infection at the Rsvl and Rpvl region in soybean. Genome 45:592-599.

Grant, M. R., Godiard, L., Straube, E., Ashfield, T., Lewald, J., Sattler, A., Innes, R. W., and Dangl, J. L. 1995. Structure of the Arabidopsis RPMI gene enabling dual specificity disease resistance. Science 269:843-846.

Gunduz, I., Buss, G. R., Chen, P., and Tolin, S. A. 2004. Genetic and phenotypic analysis of Soybean mosaic virus resistance in PI 88788 soybean. Phytopathology 94:687-692.

Hajimorad, M. R., and Hill, J. H. 2001. Rsv1-mediated resistance against Soybean mosaic virus- $\mathrm{N}$ is hypersensitive response-independent at inoculation site, but has the potential to initiate a hypersensitive response-like mechanism. Mol. Plant-Microbe Interact. 14:587-598.

Hajimorad, M. R., Eggenberger, A. L., and Hill, J. H. 2003. Evolution of Soybean mosaic virus-G7 molecularly cloned genome in Rsv1-genotype soybean results in emergence of a mutant capable of evading Rsv1mediated recognition. Virology 314:497-509.

Hajimorad, M. R., Eggenberger, A. L., and Hill, J. H. 2005. Loss and gain of elicitor function of Soybean mosaic virus G7 provoking Rsv1-mediated lethal systemic hypersensitive response maps to P3. J. Virol. 79:1215-1222.

Hajimorad, M. R., Eggenberger, A. L., and Hill, J. H. 2006. Strain-specific P3 of Soybean mosaic virus elicits Rsv1-mediated extreme resistance, but absence of P3 elicitor function alone is insufficient for virulence on Rsv1-genotype soybean. Virology 345:156-166.

Hajimorad, M. R., Eggenberger, A. L., and Hill, J. H. 2008. Adaptation of Soybean mosaic virus avirulent chimeras containing P3 sequences from virulent strains to $R s v 1$-genotype soybeans is mediated by mutations in HC-Pro. Mol. Plant-Microbe Interact. 21:937-946.

Hajimorad, M. R., Wen, R.-H., Eggenberger, A. L., Hill, J. H., and Saghai Maroof, M. A. 2011. Experimental adaptation of an RNA virus mimics natural evolution. J. Virol. 85:2557-2564.

Harrison, B. D. 2002. Virus variation in relation to resistance-breaking in plants. Euphytica 124:181-192.

Hayes, A. J., Jeong, S. C., Gore, M. A., Yu, Y. G., Buss, G. R., Tolin, S. A., and Saghai Maroof, M. A. 2004. Recombination within a nucleotidebinding-site/leucine-rich-repeat gene cluster produces new variants conditioning resistance to soybean mosaic virus in soybeans. Genetics 166:493-503.

Innes, R. W., Ameline-Torregrosa, C., Ashfield, T., Cannon, E., Cannon, S. B., Chacko, B., Chen, N. W. G., Couloux, A., Dalwani, A., Denny, R., Deshpande, S., Egan, A. N., Glover, N., Hans, C. S., Howell, S., Ilut, D., Jackson, S., Lai, H., Mammadov, J., Martin del Campo, S. M., Metcalf, M., Nguyen, A., O'Bleness, M., Pfeil, B. E., Podicheti, R., Ratnaparkhe, M. B., Samain, S., Sanders, I., Ségurens, B., Sévignac, M., Sherman-Broyles, S., Thareau, V., Tucker, D. M., Walling, J., Wawrzynski, A., Yi, J., Doyle, J. J., Geffroy, V., Roe, B. A., Saghai Maroof, M. A., and Young, N. D. 2008. Differential accumulation of retroelements and diversification of NB-LRR disease resistance genes in duplicated regions following polyploidy in the ancestor of soybean. Plant Physiol. 148:1740-1759.

Janzac, B., Fabre, F., Palloix, A., and Moury, B. 2009. Constraints on evolution of virus avirulence factors predict the durability of corresponding plant resistances. Mol. Plant Pathol. 10:599-610.

Janzac, B., Montarry, J., Palloix, A., Navaud, O., and Moury, B. 2010. A point mutation in the polymerase of Potato virus $Y$ confers virulence toward the Pvr4 resistance of pepper and a high competitiveness cost in susceptible cultivar. Mol. Plant-Microbe Interact. 23:823-830.

Jenner, C. E., Sanchez, F., Nettleship, S. B., Foster, G. D., Ponz, F., and Walsh, J. A. 2000. The cylindrical inclusion gene of Turnip mosaic virus encodes a pathogenic determinant to the Brassica resistance gene TuRB01. Mol. Plant-Microbe Interact. 13:1102-1108.

Jenner, C. E., Tomimura, K., Ohshima, K., Hughes, S. L., and Walsh, J. A. 2002. Mutations in Turnip mosaic virus P3 and cylindrical inclusion proteins are separately required to overcome two Brassica napus resistance genes. Virology 300:50-59.

Jones, J. D. G., and Dangl, J. L. 2006. The plant immune system. Nature 444:323-329.

Khatabi, B., Fajolu, O. L., Wen, R.-H., and Hajimorad, M. R. 2012. Evaluation of North American isolates of Soybean mosaic virus for gain of virulence on $R s v$-genotype soybeans with special emphasis on resistance-breaking determinants on Rsv4. Mol. Plant Pathol. 13:10771088.

Kim, B. M., Suehiro, N., Natsuaki, T., Inukai, T., and Masuta, C. 2010. The P3 protein of Turnip mosaic virus can alone induce hypersensitive response-like cell death in Arabidopsis thaliana carrying TuN1. Mol. Plant-Microbe Interact. 23:144-152.

Krause-Sakate, R., Redondo, E., Richard-Forget, F., Jadao, A. S., Houvenaghel, M.-C., German-Retana, S., Pavan, M. A., Candresse, T., 
Zerbini, F. M., and Gall, O. Le. 2005. Molecular mapping of the viral determinants of systemic wilting induced by a Lettuce mosaic virus (LMV) isolate in some lettuce cultivars. Virus Res. 109:175-180.

Lee, S.-K., Song, M.-Y., Seo, Y.-S., Kim, H.-K., Ko, S., Cao, P.-J., Suh, J.P., Yi, G., Roh, J.-H., Lee, S., An, G., Hahn, T.-R., Wang, G.-L., Ronald, P., and Jeon, J.-S. 2009. Rice Pi5-mediated resistance to Magnaporthe oryzae requires the presence of two coiled-coil-nucleotide-bindingleucine-rich repeat genes. Genetics 181:1627-1638.

Mackey, D., Holt III, B. F., Wiig, A., and Dangl, J. L. 2002. Rin4 interacts with Pseudomonas syringae type III effector molecules and is required for RPM1-mediated resistance in Arabidopsis. Cell 108:743-754.

Malapi-Nelson, M., Wen, R.-H., Ownley, B. H., and Hajimorad, M. R. 2009. Co-infection of soybean with Soybean mosaic virus and Alfalfa mosaic virus results in disease synergism and alteration in accumulation level of both viruses. Plant Dis. 93:1259-1264.

Malcuit, I., Marano, M. R., Kavanagh, T. A., De Jong, W., Forsyth, A., and Baulcombe, D. C. 1999. The 25-kDa movement protein of PVX elicits $\mathrm{Nb}$-mediated hypersensitive cell death in potato. Mol. Plant-Microbe Interact. 12:536-543.

Meshi, T., Motoyoshi, F., Adachi, A., Watanabe, Y., Takamatsu, N., and Okada, Y. 1988. Two concomitant base substitutions in the putative replicase genes of tobacco mosaic virus confer the ability to overcome the effects of a tomato resistance gene, Tm-1. EMBO (Eur. Mol. Biol. Organ.) J. 7:1575-1581.

Meshi, T., Motoyoshi, F., Maeda, T., Yoshiwoka, S., Watanabe, H., and Okada, Y. 1989. Mutations in the tobacco mosaic virus $30-\mathrm{kD}$ protein gene overcome Tm-2 resistance in tomato. Plant Cell 1:515-522.

Moon, J.-K., Jeong, S.-C., Van, K., Saghai Maroof, M. A., and Lee, S.-H. 2009. Marker-assisted identification of resistance genes to soybean mosaic virus in soybean lines. Euphytica 169:375-385.

Narusaka, M., Shirasu, K., Noutoshi, Y., Kubo, Y., Shiraishi, T., Iwabuchi, M., and Narusaka, Y. 2009. RRS1 and RPS4 provide a dual resistancegene system against fungal and bacterial pathogens. Plant J. 60:218226 .

Nirmala, J., Drader, T., Lawrence, P. K., Yin, C., Hulbert, S., Steber, C. M., Steffenson, B. J., Szabo, L. J., Wettstein, D. V., and Kleinhofs, A. 2011. Concerted action of two avirulent spore effectors activates reaction to Puccinia graminis 1 (Rpg1)-mediated cereal stem rust resistance. Proc. Natl. Acad. Sci. U.S.A. 108:14676-14681.

Peart, J. R., Mestre, P., Lu, R., Malcuit, I., and Baulcombe, D. C. 2005. NRG1, a CC-NB-LRR protein, together with N, a TIR-NB-LRR protein, mediates resistance against tobacco mosaic virus. Curr. Biol. 15:968-973.

Pfitzner, U. M., and Pfitzner, A. J. P. 1992. Expression of a viral avirulence gene in transgenic plants is sufficient to induce the hypersensitive defense reaction. Mol. Plant-Microbe. Interact. 5:318-321.

Saghai Maroof, M. A., Tucker, D. M., Skoneczka, J. A., Bowman, B. C., Tripathy, S., and Tolin, S. A. 2010. Fine mapping and candidate gene discovery of the soybean mosaic virus resistance gene, Rsv4. Plant Genome 3:14-22.

Schmutz, J., Cannon, S. B., Schlueter, J., Ma, J., Mitros, T., Nelson, W., Hyten, D. L., Song, Q., Thelen, J. J., Cheng, J., Xu, D., Hellsten, U., May, G. D., Yu, Y., Sakurai, T., Umezawa, T., Bhattacharyya, M. K., Sandhu, D., Valliyodan, B., Lindquist, E., Peto, M., Grant, D., Shu, S., Goodstein, D., Barry, K., Futrell-Griggs, M., Abernathy, B., Du, J., Tian, Z., Zhu, L., Gill, N., Joshi, T., Libault, M., Sethuraman, A., Zhang, X. C., Shinozaki, K., Nguyen, H. T., Wing, R. A., Cregan, P., Specht, J., Grimwood, J., Rokhsar, D., Stacey, G., Shoemaker, R. C., and Jackson, S. A. 2010. Genome sequence of the palaeopolyploid soybean. Nature 463:178-183.

Seo, J.-K., Lee, S.-H., and Kim, K.-H. 2009a. Strain-specific cylindrical inclusion protein of Soybean mosaic virus elicits extreme resistance and a lethal systemic hypersensitive response in two resistant soybean cultivars. Mol. Plant-Microbe. Interact. 22:1151-1159.

Seo, J.-K., Ohshima, K., Lee, H.-G., Son, M., Choi, H.-S., Lee, S.-H., Sohn, S.-H., and Kim, K.-H. 2009b. Molecular variability and genetic structure of the population of Soybean mosaic virus based on the analysis of complete genome sequences. Virology 393:91-103.

Shaner, G., Stromberg, E. L., Lacy, G. H., Barker, K. R., and Pirone, T. P. 1992. Nomenclature and concepts of pathogenicity and virulence. Annu. Rev. Phytopathol. 30:47-66

Sinapidou, E., Williams, K., Nott, L., Bahkt, S., Tör, M., Crute, I., BittnerEddy, P., and Beynon, J. 2004. Two TIR:NB:LRR genes are required to specify resistance to Peronospora parasitica isolate Cala2 in Arabidopsis. Plant J. 38:898-909.

Suh, S. J., Bowman, B. C., Jeong, N., Yang, K., Kastl, C., Tolin, S. A., Saghai Maroof, M. A., and Jeong, S.-C. 2011. The Rsv3 locus conferring resistance to Soybean mosaic virus is associated with a cluster of coiled-coil nucleotide-binding leucine-rich repeat genes. Plant Genome 4:55-64.

Urcuqui-Inchima, S., Haenni, A.-L., and Bernardi, F. 2001. Potyvirus proteins: A wealth of functions. Virus Res. 74:157-175.

Viel, C., Ide, C., Cui, X., Wang, A., Farsi, M., Michelutti, R., and Strömvik, M. 2009. Isolation, partial sequencing, and phylogenetic analyses of Soybean mosaic virus (SMV) in Ontario and Quebec. Can J. Plant Pathol. 31:108-113.

Vijayapalani, P., Maeshima, M., Nagasaki-Takekuchi, N., and Miller, W. A. 2012. Interaction of the trans-frame potyvirus protein P3N-PIPO with host protein PCaP1 facilitates potyvirus movement. PLoS Pathog. 8:e1002639.

Wang, L., Eggenberger, A., Hill, J., and Bogdanove, A. J. 2006. Pseudomonas syringae effector $a v r B$ confers soybean cultivar-specific avirulence on Soybean mosaic virus adapted for transgene expression but effector avrPto does not. Mol. Plant-Microbe Interact. 19:304-312.

Wen, R.-H., and Hajimorad, M. R. 2010. Mutational analysis of the putative pipo of soybean mosaic virus suggests disruption of PIPO protein impedes movement. Virology 400:1-7.

Wen, R.-H., Saghai Maroof, M. A., and Hajimorad, M. R. 2011. Amino acid changes in P3, and not the overlapping pipo-encoded protein, determine virulence of Soybean mosaic virus on functionally immune Rsv1-genotype soybean. Mol. Plant Pathol. 12:799-807.

Yu, Y. G., Buss, G. R., and Saghai Maroof, M. A. 1996. Isolation of a superfamily of candidate disease-resistance genes in soybean based on a conserved nucleotide-binding site. Proc. Natl. Acad. Sci. U.S.A. 93:11751-11756.

Zhang, C., Hajimorad, M. R., Eggenberger, A. L., Tsang, S., Whitham, S. A., and Hill, J. H. 2009. Cytoplasmic inclusion cistron of Soybean mosaic virus serves as a virulence determinant on Rsv3-genotype soybean and a symptom determinant. Virology 391:240-248.

Zhang, C., Grosic, S., Whitham, S. A., and Hill, J. H. 2012. The requirement of multiple defense genes in soybean Rsv1 mediated extreme resistance to Soybean mosaic virus. Mol. Plant-Microbe. Interact. 25:1307-1313.

Zhang, X., Feng, Y., Cheng, H., Tian, D., Yang, S., and Chen, J.-Q. 2011. Relative evolutionary rates of NBS-encoding genes revealed by soybean segmental duplication. Mol. Genet. Genomics 285:79-90. 